\title{
Risk factors and risk prediction models for colorectal cancer metastasis and recurrence: an umbrella review of systematic reviews and meta-analyses of observational studies
}

Wei Xu1, Yazhou He1, Yuming Wang ${ }^{2}$, Xue Li', Jane Young ${ }^{3}$, John P. A. loannidis ${ }^{4,5,6,7,8}$, Malcolm G. Dunlop ${ }^{9,10}$ and Evropi Theodoratou ${ }^{1,10^{*}}$ (i)

\begin{abstract}
Background: There is a clear need for systematic appraisal of models/factors predicting colorectal cancer (CRC) metastasis and recurrence because clinical decisions about adjuvant treatment are taken on the basis of such variables.

Methods: We conducted an umbrella review of all systematic reviews of observational studies (with/without metaanalysis) that evaluated risk factors of CRC metastasis and recurrence. We also generated an updated synthesis of risk prediction models for CRC metastasis and recurrence. We cross-assessed individual risk factors and risk prediction models.
\end{abstract}

Results: Thirty-four risk factors for CRC metastasis and 17 for recurrence were investigated. Twelve of 34 and 4/17 risk factors with $p<0.05$ were estimated to change the odds of the outcome at least 3-fold. Only one risk factor (vascular invasion for lymph node metastasis [LNM] in pT1 CRC) presented convincing evidence. We identified 24 CRC risk prediction models. Across 12 metastasis models, six out of 27 unique predictors were assessed in the umbrella review and four of them changed the odds of the outcome at least 3-fold. Across 12 recurrence models, five out of 25 unique predictors were assessed in the umbrella review and only one changed the odds of the outcome at least 3-fold.

Conclusions: This study provides an in-depth evaluation and cross-assessment of 51 risk factors and 24 prediction models. Our findings suggest that a minority of influential risk factors are employed in prediction models, which indicates the need for a more rigorous and systematic model construction process following evidence-based methods.

Keywords: Colorectal cancer, Metastasis, Recurrence, Risk factors, Risk prediction models, Umbrella review

\footnotetext{
* Correspondence: E.Theodoratou@ed.ac.uk

'Centre for Global Health, Usher Institute, University of Edinburgh, Edinburgh EH8 9AG, UK

${ }^{10}$ Edinburgh Cancer Research Centre, Institute of Genetics and Molecular Medicine, University of Edinburgh, Edinburgh EH4 2XU, UK

Full list of author information is available at the end of the article
}

(c) The Author(s). 2020 Open Access This article is licensed under a Creative Commons Attribution 4.0 International License, which permits use, sharing, adaptation, distribution and reproduction in any medium or format, as long as you give appropriate credit to the original author(s) and the source, provide a link to the Creative Commons licence, and indicate if changes were made. The images or other third party material in this article are included in the article's Creative Commons licence, unless indicated otherwise in a credit line to the material. If material is not included in the article's Creative Commons licence and your intended use is not permitted by statutory regulation or exceeds the permitted use, you will need to obtain permission directly from the copyright holder. To view a copy of this licence, visit http://creativecommons.org/licenses/by/4.0/ The Creative Commons Public Domain Dedication waiver (http://creativecommons.org/publicdomain/zero/1.0/) applies to the data made available in this article, unless otherwise stated in a credit line to the data. 


\section{Background}

Around $20-25 \%$ of patients with colorectal cancer (CRC) present with metastasis at initial diagnosis, while patients who are apparently cancer-free on investigation at diagnosis subsequently develop locoregional recurrence (18\%), distant (78\%) recurrence, or both (4\%) [1]. Metastasis occurs when cancer cells from the original tumor are able to proliferate in local, regional, or distant tissues; lymph nodes; or organs via lymphatic, blood, or even transcoelomic spread [2]. CRC recurrence is defined as local, regional, and distant metastatic recurrence after a diseasefree period [3]. Local recurrence refers to CRC relapse that occurs at the site of original surgical resection [4], while regional recurrence occurs at draining lymph nodes and/or lateral pelvic lymph nodes [3]. Distant metastatic recurrence involves the liver (accounts for $40-50 \%$ of metastases), the lung (accounts for $10-20 \%$ of metastases), the peritoneum, the ovaries, the adrenal glands, the bone, and the brain $[1,5]$. It is estimated that 5 -year survival rates are around $90 \%, 70 \%$, and $10 \%$ for CRC localized, regional, and distant metastatic stages [6].

Validating individual risk factors and even more so multivariable prediction models of multiple risk factors for local, regional, or distant metastasis and recurrence is crucially important as these could guide management of the primary tumor and provide prognostic information for patients and their cancer clinicians. Prediction models may be more successful if they consider the most informative factors. This knowledge may eventually prove useful in managing CRC treatment with betterinformed patient choices. Understanding the underlying validity and predictive performance of risk factors for locoregional recurrence is particularly relevant, given progressive moves towards organ-preserving approaches such as endoscopic resection (EMR), trans-anal microscopic surgery (TEMS), and neo-adjuvant chemoradiotherapy for rectal cancer [1], since organ preservation may be at the expense of elevated recurrence rates. The corollary also applies since the risk-benefit ratio of extensive locoregional surgery and/or radiotherapy may be detrimentally impacted by future distant metastases.

A number of systematic reviews (with/without metaanalyses) have investigated existing risk factors for CRC metastasis and recurrence [7-10]. However, there is a need for a comprehensive evaluation of the available epidemiological evidence. Here, we conducted an umbrella review to identify and evaluate associations between risk factors and risk of CRC metastasis and recurrence. We also systematically collected and evaluated predictive models on CRC prognostic outcomes. We then conducted a comparative cross-assessment between the identified risk factors and the predictors employed in risk prediction models to examine to what extent predictive models include the most influential factors.

\section{Methods}

\section{Protocol}

The study protocol was developed in accordance with the reporting guidance in the Preferred Reporting Items for Systematic Reviews and Meta-Analyses Protocols (PRISMA-P) statement [11].

\section{Umbrella review of systematic reviews (with/without meta-analyses) of risk factors \\ Literature search and eligibility criteria}

A systematic search was performed in PubMed, Cochrane Library (Wiley), Web of Science (Thomson Reuters), and EMBASE (Ovid) from inception to 7 October 2019, to identify systematic reviews of observational studies with or without a meta-analysis that evaluated the associations between risk factors and risk of metastasis and recurrence in CRC (Additional file 1: Table S1). We further hand-searched reference lists of the retrieved eligible publications to identify additional relevant studies. All identified publications went through a parallel review of the title, abstract, and full text (performed by WX and YM independently) based on pre-defined inclusion and exclusion criteria following "PICOS." In particular, we included human participants from observational studies with no restriction to settings. Conversely, animal, in vitro, and in vivo experiments were excluded. For study outcomes, we included CRC metastasis (local, regional, or distant metastasis in tissues, lymph nodes, or organs at diagnosis) and CRC recurrence (local, regional, or distant metastatic recurrence in tissues, lymph nodes, or organs after a disease-free period). For study design, we included systematic reviews of observational studies with or without meta-analysis. Conversely, literature reviews, individual observational studies, systematic reviews, and metaanalyses that investigated the evidence on the efficacy of pharmaceutical drugs and therapeutic procedures were excluded. We included publications in peer-reviewed journals, and therefore, gray literature, comments, conference abstracts, and interviews were excluded.

\section{Data extraction}

Data were extracted by one investigator (WX) and checked by a second investigator $(\mathrm{YH})$. For each included meta-analysis, the following items were extracted: study citation details, number of studies included, study design, study population, number of events and size of total population, risk factors, outcomes examined, reported summary meta-analytic estimates (e.g., risk ratio $[R R]$, odds ratio $[\mathrm{OR}]$, hazard ratio $[\mathrm{HR}]$, the corresponding 95\% confidence interval [95\% CI], $p$ value, and heterogeneity measures), instrument applied for quality and risk of bias assessment of component studies, and quality assessment result. The following items were 
further extracted from the individual component studies: study citation details, study design, study population, risk factors, outcomes examined, number of events and size of total population in exposed and unexposed groups, effect size, and 95\% CI.

\section{Evidence synthesis and evaluation}

First, when two or more meta-analyses examining associations between the same risk factor and the same outcome were identified, the most recent meta-analysis of prospective cohort studies with the largest event number was prioritized and retained for further analysis. We also compared whether the results reported in overlapping meta-analyses were concordant in terms of direction, statistical significance, and association magnitude.

Second, we estimated the following metrics for each unique meta-analysis: (1) The summary effect size along with 95\% CI was estimated based on random-effects models (DerSimonian and Laird (DL)) when the number of component studies was five or more and the Hartung-Knapp-Sidik-Jonkman (HKSJ) when the number of component studies was less than five [12, 13]. (2) Heterogeneity was assessed by the $I^{2}$ statistic [14]. (3) The 95\% prediction interval was estimated. (4) The small study effect was estimated by Egger's regression asymmetry test [15]. (5) Excess significance was assessed by a chi-square test [16]. Based on these metrics and by applying a set of pre-defined criteria (Additional file 1: Table S8), we evaluated the credibility of the evidence for each risk factor and categorized the evidence as convincing, highly suggestive, suggestive, or weak $[17,18]$.

Lastly, for all meta-analyses that statistically represented at least 3-fold changes in the odds of the outcome, we evaluated the methodological quality and risk of bias based on the Assessment of Multiple Systematic Reviews 2.0 (AMSTAR 2.0) checklist [19]. We used an odds ratio of 3.0 as a threshold for what is a substantially large effect. There is no consensus on what an optimal threshold might be, but values between 2 and 5 are proposed typically [20].

\section{Sensitivity analysis}

We re-ran all meta-analyses by evaluating the outcome definitions of each individual component study reclassifying the outcomes to (i) CRC metastasis at presentation,

(ii) $\mathrm{CRC}$ local recurrence after a disease-free period, and (iii) CRC distant recurrence after a disease-free period.

\section{Comparative cross-assessment of risk factors and risk prediction models}

We performed a comparative cross-assessment between risk factors evaluated in the umbrella review and risk predictors included in existing prediction models. A recently published systematic review conducted by our team [21] investigated a total number of 15 prediction models for prediction of metastasis and recurrence in CRC patients with surgical resection (metastasis: $N=6$; recurrence: $N=9$ ). We updated the original search to identify studies developing and/or validating risk prediction models to predict metastasis and recurrence in all CRCs, with no restriction on whether the tumor was resected. We performed a systematic search in PubMed from inception to 7 October 2019 to identify eligible studies. We extracted data relevant to study design, study population, prediction outcome, prediction time horizon, predictors, model performance, and model presentation from each included study. We created a catalog of all variables that had been included across CRC metastasis prognostic models and separately across CRC recurrence prognostic models (presented in the same order as in the respective tables). We then assessed whether the included risk predictors were evaluated or not in the umbrella review described above. If yes, we also recorded the magnitude of the summary relative risk (typically odds ratio) and noted how many of those represented at least 3 -fold changes in the odds of the outcome and how many had convincing or highly suggestive evidence in our assessment.

All statistical analyses were conducted in Stata, version 14.0 (StataCorp), and R, version 3.3.0 (R Foundation for Statistical Computing).

\section{Results}

\section{Literature review}

A total of 2033 publications were retrieved from the systematic search in four databases. Eventually, 43 publications met all inclusion criteria (Fig. 1, Additional file 1: Table S2) and that included 9 systematic reviews (metastasis: $N=7$; recurrence: $N=2$ ) and 81 meta-analyses (metastasis: $N=61$; recurrence: $N=20$; Additional file 1: Table S3 and Table S4) of observational studies. A total of 18 overlapping meta-analyses that examined associations between the same risk factor and the same outcome were identified (Additional file 1: Table S5). The most recent meta-analysis with the largest event number was prioritized. Within the remaining 63 unique metaanalyses, 12 meta-analyses from four publications did not report detailed OR, RR, or HR in forest plots. Finally, 51 unique meta-analyses were retained for analysis, which reported 34 unique risk factors for CRC metastasis and 17 risk factors for recurrence (Additional file 1: Table S6 and Table S7).

\section{Meta-analyses of risk factors for CRC metastasis}

Overall, 61 eligible meta-analyses of observational studies investigating risk factors for CRC metastasis were identified (Additional file 1: Table S3). More than one meta-analysis was conducted for seven risk factors 


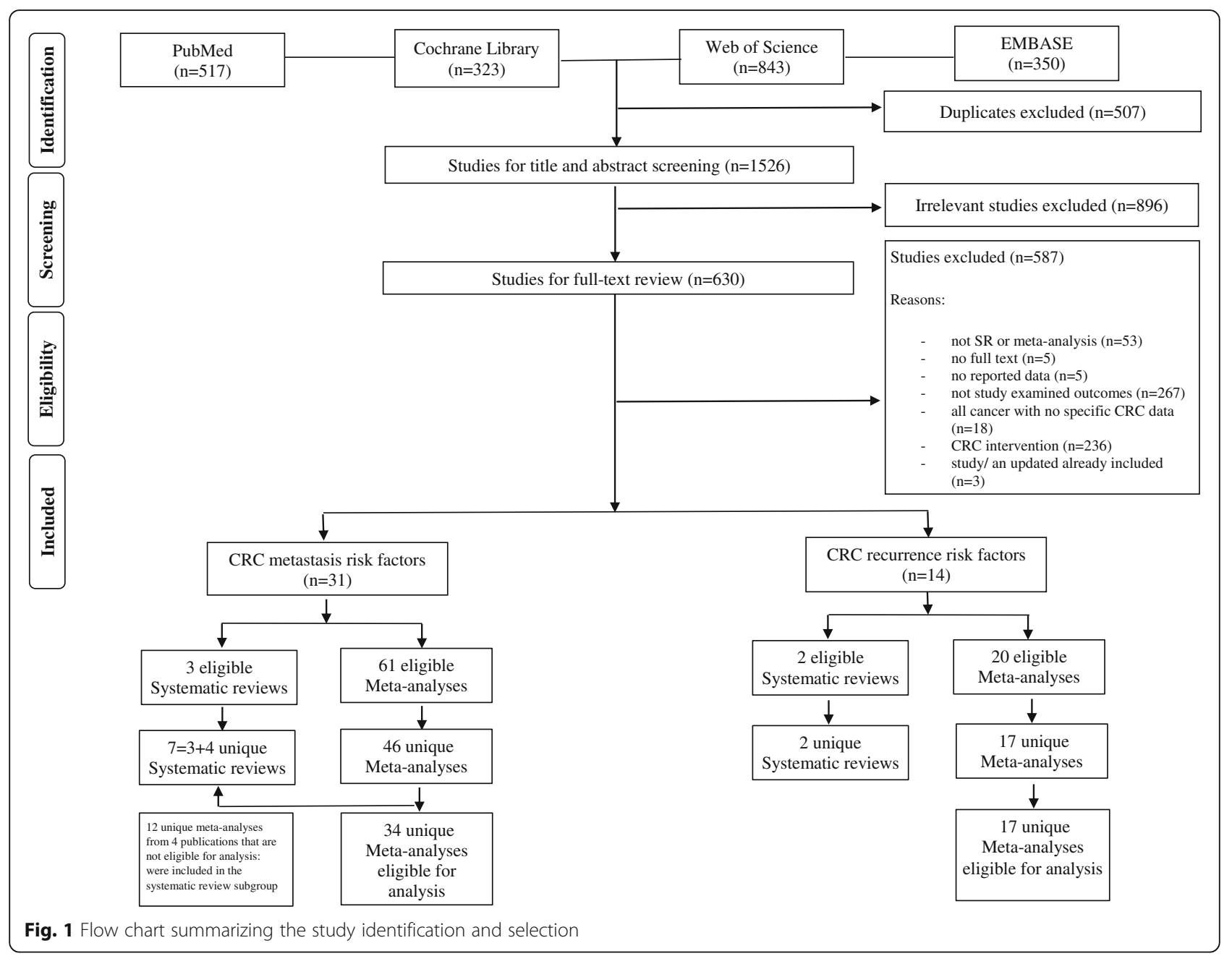

(Additional file 1: Table S5). The direction of the summary effect size and the presence of nominal statistical significance $(p<0.05)$ of the reported associations in overlapping meta-analyses were concordant for six (86\%) risk factors (Additional file 1: Table S5).

A total of 34 unique meta-analyses with available data were retained for further analysis (Additional file 1: Table S6). The median number of included component studies was five (range 2-41), the median number of the total population was 983 (range 76-10,128), and the median number of events was 138 (range 16-1808). The meta-analyses reported a wide range of risk factors (Additional file 1: Table S6): 17 histopathological risk factors (50\%), 13 biomarkers (38\%), three genetic risk factors (9\%), and one demographic risk factor (3\%). Overall, 21 (62\%) of 34 unique meta-analyses reported effect sizes at $p<0.05$ (Table 1). Based on the predefined credibility criteria, only one (3\%) histopathological risk factor (vascular invasion for LNM in pT1 $C R C$ ) presented convincing evidence (see Additional file 1: Table S9 for the credibility assessment of all identified risk factors). Furthermore, 12 of 21 probed risk factors with $p<0.05$ had an effect size suggesting $\geq 3$-fold change in the odds of the outcome, while this was also seen for the point estimates in four of 13 probed risk factors where the meta-analysis had $p \geq 0.05$ (Table 1 ).

\section{Meta-analyses of risk factors for CRC recurrence}

Overall, 20 eligible meta-analyses of observational studies investigating risk factors for CRC recurrence were identified (Additional file 1: Table S4). More than one meta-analysis was conducted for three risk factors (Additional file 1: Table S5). The direction of the summary effect size and the presence of nominal statistical significance $(p<0.05)$ of the reported associations between the same risk factor and the same outcome in overlapping meta-analyses were concordant for two $(67 \%)$ risk factors (Additional file 1: Table S5).

A total of 17 unique meta-analyses with available data were retained for further analysis (Additional file 1: Table S7). The median number of included component studies was six (range 2-26), the median number of the 
Table 1 Summary of evidence credibility assessment of 34 unique meta-analyses of observational studies investigating the associations between risk factors and CRC metastasis

\begin{tabular}{|c|c|c|c|c|c|}
\hline Population & Outcome & Risk factor & Risk factor prevalence & Effect size $(95 \% \mathrm{Cl})$ & Evidence classificatio \\
\hline \multicolumn{6}{|c|}{ Histopathological risk factor } \\
\hline pT1 CRC & Lymph node metastasis in pT1 CRC & Vascular invasion & $330 / 1731=19 \%$ & $2.73(1.98-3.78)$ & Convincing \\
\hline pT1 CRC & Lymph node metastasis in pT1 CRC & Lymphatic invasion & $906 / 3347=27 \%$ & $6.78(5.29-8.69)$ & Highly suggestive \\
\hline pT1 CRC & Lymph node metastasis in pT1 CRC & Tumor budding & $2401 / 10,128=24 \%$ & $6.39(5.23-7.80)$ & Highly suggestive \\
\hline CRC & Lymph node metastasis in CRC & Tumor budding & $1955 / 6739=29 \%$ & $4.96(3.97-6.19)$ & Highly suggestive \\
\hline Rectal cancer & $\begin{array}{l}\text { Lymph node metastasis in rectal } \\
\text { cancer }\end{array}$ & Tumor size $>1 \mathrm{~cm}$ & $203 / 348=58 \%$ & $6.76(3.25-14.04)$ & Highly suggestive \\
\hline pT1 CRC & Lymph node metastasis in pT1 CRC & $\begin{array}{l}\text { Lymphovascular } \\
\text { invasion }\end{array}$ & $340 / 1695=20 \%$ & $4.81(3.14-7.36)$ & Suggestive \\
\hline pT1 CRC & $\begin{array}{l}\text { Lymph node metastasis in pT1 CRC } \\
\text { patients who underwent additional } \\
\text { surgeries after an endoscopic } \\
\text { resection }\end{array}$ & $\begin{array}{l}\text { Lymphovascular } \\
\text { invasion }\end{array}$ & $91 / 313=29 \%$ & $5.29(2.34-11.98)$ & Suggestive \\
\hline pT1 CRC & Lymph node metastasis in pT1 CRC & Poor differentiation & $94 / 2722=4 \%$ & $5.61(2.90-10.83)$ & Suggestive \\
\hline Rectal cancer & $\begin{array}{l}\text { Lymph node metastasis in rectal } \\
\text { cancer }\end{array}$ & $\begin{array}{l}\text { Muscularis properia } \\
\text { invasion }\end{array}$ & $122 / 322=38 \%$ & $5.08(2.32-11.11)$ & Suggestive \\
\hline pT1 CRC & Lymph node metastasis in pT1 CRC & $\begin{array}{l}\text { Submucosal invasion } \\
\geq 1 \mathrm{~mm}\end{array}$ & $2389 / 2922=82 \%$ & $2.95(1.39-6.27)$ & Weak \\
\hline Small rectal NETs & $\begin{array}{l}\text { Lymph node metastasis in small } \\
\text { rectal NETs treated by local excision }\end{array}$ & $\begin{array}{l}\text { Lymphovascular } \\
\text { invasion }\end{array}$ & $104 / 517=20 \%$ & $5.02(1.16-21.72)$ & Weak \\
\hline Rectal cancer & $\begin{array}{l}\text { Lymph node metastasis in rectal } \\
\text { cancer }\end{array}$ & Central depression & $32 / 76=42 \%$ & $3.00(2.10-4.28)$ & Weak \\
\hline Rectal cancer & $\begin{array}{l}\text { Synchronous metastasis in rectal } \\
\text { cancer }\end{array}$ & $\begin{array}{l}\text { MRI-detected } \\
\text { extramural vascular } \\
\text { invasion (mrEMVI) }\end{array}$ & $212 / 804=26 \%$ & $5.65(2.12-15.05)$ & Weak \\
\hline Small rectal NETs & $\begin{array}{l}\text { Lymph node metastasis in small } \\
\text { rectal NETs treated by local excision }\end{array}$ & Lymphatic invasion & $73 / 493=15 \%$ & $5.54(0.02-1752.46)$ & No association \\
\hline Rectal cancer & $\begin{array}{l}\text { Lymph node metastasis in rectal } \\
\text { cancer }\end{array}$ & Vascular invasion & $46 / 168=27 \%$ & $5.86(0.77-44.62)$ & No association \\
\hline Small rectal NETs & $\begin{array}{l}\text { Lymph node metastasis in small } \\
\text { rectal NETs treated by local excision }\end{array}$ & Vascular invasion & $75 / 211=36 \%$ & $3.63(0.05-268.57)$ & No association \\
\hline pT1 CRC & $\begin{array}{l}\text { Lymph node metastasis in pT1 CRC } \\
\text { patients who underwent additional } \\
\text { surgeries after an endoscopic } \\
\text { resection }\end{array}$ & $\begin{array}{l}\text { Poor or moderate } \\
\text { differentiation }\end{array}$ & $122 / 209=58 \%$ & $3.77(1.12-123.16)$ & No association \\
\hline \multicolumn{6}{|l|}{ Biomarker } \\
\hline CRC & Lymph node metastasis in CRC & $\begin{array}{l}\text { Downregulated E- } \\
\text { cadherin expression }\end{array}$ & $829 / 1573=53 \%$ & $0.49(0.34-0.72)$ & Highly suggestive \\
\hline CRC & Hepatic metastasis (distant) in CRC & Circulating tumor cells & $103 / 310=33 \%$ & $6.38(2.67-15.26)$ & Suggestive \\
\hline CRC & Lymph node metastasis in CRC & $\begin{array}{l}\text { Low MUC2 expression } \\
\text { level }\end{array}$ & $613 / 1335=46 \%$ & $1.42(1.19-1.69)$ & Suggestive \\
\hline CRC & Distant metastasis in CRC & $\begin{array}{l}\text { Downregulated E- } \\
\text { cadherin expression }\end{array}$ & $509 / 1027=50 \%$ & $0.45(0.23-0.91)$ & Weak \\
\hline CRC & Lymph node metastasis in CRC & Circulating tumor cells & $797 / 1802=44 \%$ & $1.62(1.17-2.23)$ & Weak \\
\hline CRC & Lymph node metastasis in CRC & p16 protein expression & $482 / 800=60 \%$ & $0.50(0.30-0.84)$ & Weak \\
\hline CRC & Distant metastasis in CRC & $\begin{array}{l}\text { Cyclin D1 } \\
\text { overexpression }\end{array}$ & $952 / 1515=63 \%$ & $0.60(0.36-0.99)$ & Weak \\
\hline CRC & Distant metastasis in CRC & $\begin{array}{l}\beta \text {-catenin } \\
\text { overexpression in the } \\
\text { nucleus }\end{array}$ & $283 / 531=53 \%$ & $0.48(0.29-0.79)$ & Weak \\
\hline CRC & Lymph node metastasis in CRC & CD147 expression & $603 / 815=74 \%$ & $1.41(0.39-5.01)$ & No association \\
\hline
\end{tabular}


Table 1 Summary of evidence credibility assessment of 34 unique meta-analyses of observational studies investigating the associations between risk factors and CRC metastasis (Continued)

\begin{tabular}{|c|c|c|c|c|c|}
\hline Population & Outcome & Risk factor & Risk factor prevalence & Effect size $(95 \% \mathrm{Cl})$ & Evidence classification \\
\hline CRC & Distant metastasis in CRC & CD147 expression & $405 / 538=75 \%$ & $\begin{array}{l}2.32(1.34 \mathrm{E}-06 \text { to } \\
4.03 \mathrm{E}+06)\end{array}$ & No association \\
\hline CRC & Lymph node metastasis in CRC & CD133 expression & $550 / 1629=34 \%$ & $1.15(0.82-1.62)$ & No association \\
\hline CRC & Distant metastasis in CRC & CD133 expression & $300 / 1064=28 \%$ & $1.54(0.39-6.09)$ & No association \\
\hline CRC & Lymph node metastasis in CRC & $\begin{array}{l}\text { HER-2 } \\
\text { immunohistochemical } \\
\text { expression }\end{array}$ & $440 / 1289=34 \%$ & $1.90(0.90-4.02)$ & No association \\
\hline \multicolumn{6}{|c|}{ Genetic risk factor } \\
\hline CRC & Lymph node metastasis in CRC & BRAF mutation & $736 / 1142=64 \%$ & $0.75(0.49-1.14)$ & No association \\
\hline CRC & Lymph node metastasis in CRC & $\begin{array}{l}\text { RASSF1A promoter } \\
\text { methylation }\end{array}$ & $100 / 184=54 \%$ & $1.61(0.16-16.16)$ & No association \\
\hline CRC & Distant metastasis in CRC & $\begin{array}{l}\text { RASSF1A promoter } \\
\text { methylation }\end{array}$ & $153 / 417=37 \%$ & $2.57(0.64-10.24)$ & No association \\
\hline \multicolumn{6}{|c|}{ Demographic risk factor } \\
\hline pT1 CRC & Lymph node metastasis in pT1 CRC & Female gender & $465 / 1329=35 \%$ & $2.23(0.78-6.42)$ & No association \\
\hline
\end{tabular}

Abbreviation: $\mathrm{Cl}$ confidence interval, $C R C$ colorectal cancer, NET neuroendocrine tumor

total population was 2773 (range 252-39,745), and the median number of events was 551 (range 57-3675). The meta-analyses reported a wide range of risk factors (Additional file 1: Table S7): five histopathological risk factors (29\%), two biomarkers (12\%), one genetic risk factor $(6 \%)$, five clinical risk factors $(29 \%)$, one comorbidity (6\%), and three anthropometric indices (18\%). Overall, $11(65 \%)$ of 17 unique meta-analyses reported effect sizes at $p<0.05$ (Table 2).

No risk factor presented convincing evidence (Additional file 1: Table S10). In addition, four of 11 probed risk factors with $p<0.05$ had an effect size suggesting $\geq$ 3 -fold change in the odds of the outcome (Table 2).

\section{Methodological quality and risk of bias assessment}

We assessed the methodological quality of 10 publications that included 16 meta-analyses of observational studies that statistically represented at least 3-fold changes in the odds of the outcome, using the AMSTAR 2.0 checklist (Additional file 1: Table S13). All assessed studies had more than one critical flaw [usually in items $2(14 / 16,88 \%), 7(16 / 16,100 \%)$, and $13(13 / 16,81 \%)]$ and several non-critical flaws [usually in items $3(11 / 16$, $69 \%), 10(16 / 16,100 \%)$, and $12(16 / 16,100 \%)]$. It should be noted that all assessed meta-analyses had critically low quality. Therefore, the credibility of the available evidence should be interpreted with caution.

\section{Sensitivity analysis of redefying the disease outcome groups}

We performed a sensitivity analysis to include individual component studies investigating risk factors for metastasis at presentation and re-evaluated the credibility of the evidence (Additional file 1: Table S11). A total of 16 unique meta-analyses including 67 (27\%) component studies were retained and investigated. The remaining 185 (73\%) studies did not illustrate when metastasis was present (i.e., at diagnosis or after a disease-free period) and therefore could not be included in this sensitivity analysis. Based on the pre-defined criteria, no risk factor presented convincing evidence.

Similarly, a sensitivity analysis was performed to include individual component studies investigating risk factors for local or distant recurrence (Additional file 1: Table S12). A total of 13 unique meta-analyses composed of 81 (58\%) component studies (including five meta-analyses investigating distant metastasis after a period of being disease-free) were retained and investigated. The remaining 59 (42\%) studies did not separate local or distant recurrence and therefore could not be included in our sensitivity analysis. Furthermore, no risk factor presented convincing evidence (Additional file 1: Table S12).

\section{Comparative cross-assessment between risk factors evaluated in the umbrella review and risk predictors applied in existing prediction models Prediction models for CRC metastasis}

Twelve prognostic models have been developed for prediction of CRC metastasis [22-33] (Table 3). The median number of included predictors was four (range 39), and 27 unique predictors were included in at least one model. Cancer stage $(N=9,75 \%)$ was the most commonly used predictor variable in the 12 prognostic models. Other common predictors included histopathological risk factors such as positive lymph nodes $(N=3$, 
Table 2 Summary of evidence credibility assessment of 17 unique meta-analyses of observational studies investigating the associations between risk factors and CRC recurrence

\begin{tabular}{|c|c|c|c|c|c|}
\hline Population & Outcome & Risk factor & Risk factor prevalence & Effect size $(95 \% \mathrm{Cl})$ & Evidence classification \\
\hline \multicolumn{6}{|c|}{ Histopathological risk factor } \\
\hline CRC & Overall recurrence in CRC & Tumor budding & $802 / 2773=29 \%$ & $5.50(3.65-8.29)$ & Highly suggestive \\
\hline CRC & Overall recurrence in CRC & Extranodal extension (ENE) & $376 / 877=43 \%$ & $2.07(1.65-2.61)$ & Highly suggestive \\
\hline Rectal cancer & Local recurrence in rectal cancer & Perineural invasion (PNI) & $336 / 1700=20 \%$ & $3.21(2.33-4.44)$ & Highly suggestive \\
\hline Rectal cancer & $\begin{array}{l}\text { Distant metastatic recurrence in } \\
\text { rectal cancer }\end{array}$ & $\begin{array}{l}\text { MRI-detected extramural vascular } \\
\text { invasion (mrEMVl) }\end{array}$ & $350 / 1262=28 \%$ & $3.91(2.61-5.86)$ & Highly suggestive \\
\hline CRC & Local recurrence in CRC & Intramural vascular invasion (IMVI) & $137 / 503=27 \%$ & $1.55(0.11-21.28)$ & No association \\
\hline \multicolumn{6}{|l|}{ Biomarker } \\
\hline CRC & Overall recurrence in CRC & $\begin{array}{l}\text { Absence of peritoneal free tumor } \\
\text { cells in pre-resection }\end{array}$ & $524 / 593=88 \%$ & $0.38(0.16-0.91)$ & Weak \\
\hline CRC & Overall recurrence in CRC & $\begin{array}{l}\text { Absence of peritoneal free tumor } \\
\text { cells in post-resection }\end{array}$ & $214 / 252=85 \%$ & $0.07(0.02-0.21)$ & Weak \\
\hline \multicolumn{6}{|c|}{ Genetic risk factor } \\
\hline CRC & Overall recurrence in CRC & PTGS2 (also known as COX-2) & $787 / 1516=52 \%$ & $2.78(1.76-4.40)$ & Suggestive \\
\hline \multicolumn{6}{|c|}{ Clinical risk factor } \\
\hline CRC & Local recurrence in CRC & Anastomotic leakage (AL) & $3929 / 39,745=10 \%$ & $1.90(1.48-2.43)$ & Suggestive \\
\hline Rectal cancer & Local recurrence in rectal cancer & Anastomotic leakage (AL) & $1300 / 13,665=10 \%$ & $1.61(1.25-2.08)$ & Suggestive \\
\hline CRC & Distant recurrence in CRC & Anastomotic leakage (AL) & $865 / 10,392=8 \%$ & $1.20(0.94-1.52)$ & No association \\
\hline Rectal cancer & Distant recurrence in rectal cancer & Anastomotic leakage (AL) & $566 / 5221=11 \%$ & $1.06(0.72-1.58)$ & No association \\
\hline Colon cancer & Local recurrence in colon cancer & Anastomotic leakage (AL) & $91 / 1990=5 \%$ & $2.19(0.55-8.68)$ & No association \\
\hline \multicolumn{6}{|l|}{ Comorbidity } \\
\hline CRC & Overall recurrence in CRC & Diabetes & $429 / 4979=9 \%$ & $1.26(0.70-2.30)$ & No association \\
\hline \multicolumn{6}{|c|}{ Anthropometric indices } \\
\hline CRC & Overall recurrence in CRC & Underweight & $2752 / 17,636=16 \%$ & $1.13(1.05-1.21)$ & Weak \\
\hline CRC & Overall recurrence in CRC & Obese & $6362 / 21,246=30 \%$ & $1.07(1.02-1.13)$ & Weak \\
\hline CRC & Overall recurrence in CRC & Overweight & $13,225 / 28,109=47 \%$ & $1.00(0.96-1.05)$ & No association \\
\hline
\end{tabular}

Abbreviation: $\mathrm{Cl}$ confidence interval, $C R C$ colorectal cancer

$25 \%)$, tumor grade or differentiation $(N=2,17 \%)$, and tumor histological type $(N=3,25 \%)$; biomarkercarcinoembryonic antigen $(\mathrm{CEA})(N=3,25 \%)$; age $(N=$ $3,25 \%)$; gender $(N=2,17 \%)$; and clinical treatment such as surgery, chemotherapy, and radiotherapy $(N=3,25 \%)$. Five models (42\%) performed internal validation, and four models (33\%) were validated in external datasets.

We conducted a cross-assessment between these predictors and 34 risk factors that were evaluated in our umbrella review. Six of 27 unique predictors (tumor budding, tumor differentiation, tumor size, vascular invasion, submucosal invasion, and sex) were evaluated in the umbrella review (Table 5). The associated ORs for these six risk factors varied from 2.23 to 6.76 , and four of them (67\%) corresponded to $\geq 3$ fold change in the odds of the outcome. Of the remaining 28 risk factors that were not employed in prediction models, ORs varied from 0.45 to 6.78 , and 13 (46\%) represented $\geq 3$-fold change in the odds of the outcome.

In addition, we compared the overlapping outcomes to investigate whether prediction models had included influential risk factors (those presented convincing evidence or with 3-fold change in the odds of the outcome) when they predicted the same outcomes as those evaluated in the umbrella review (Table 6). In total, four overlapping outcomes were found in this cross-assessment (LNM in pT1 CRC, LNM in CRC, hepatic metastasis in $C R C$, and distant metastasis in CRC). For only one outcome (LNM in pT1 CRC), two prognostic models [22, 28 ] included four risk predictors that were also evaluated in the umbrella review, two of which corresponded to $\geq$ 3 -fold change in the odds of the outcome (tumor budding, tumor differentiation).

\section{Prediction models for CRC recurrence}

Twelve prognostic models [31, 32, 34-43] were developed for prediction of CRC recurrence (Table 4). The median number of risk predictors applied in 12 prognostic models was five (range 2-8), and 25 unique predictors were included in at least one model. AJCC (TNM) cancer stage was the predictor variable $(N=7,58 \%)$ used in the majority of $\mathrm{CRC}$ recurrence risk prediction 


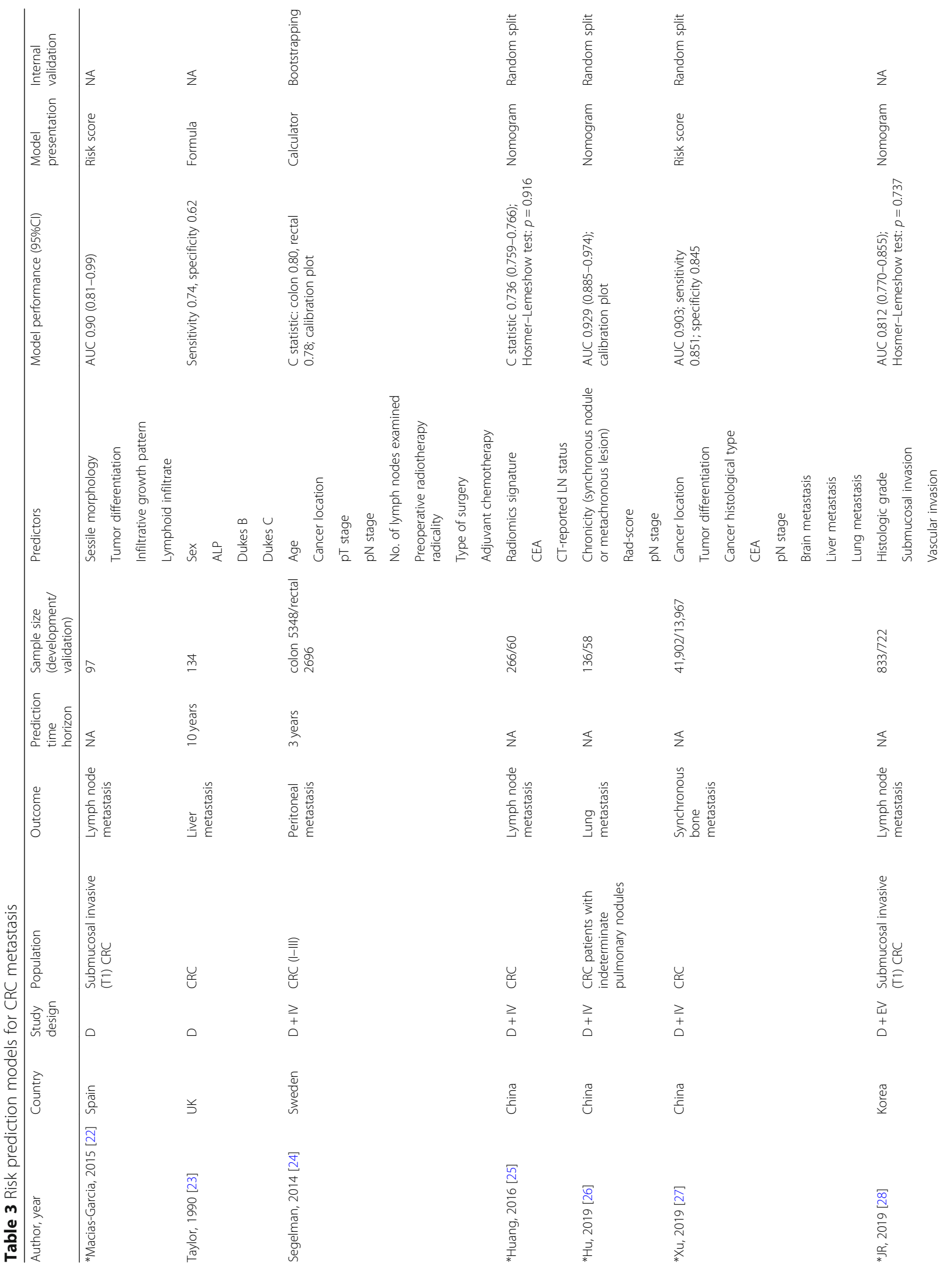




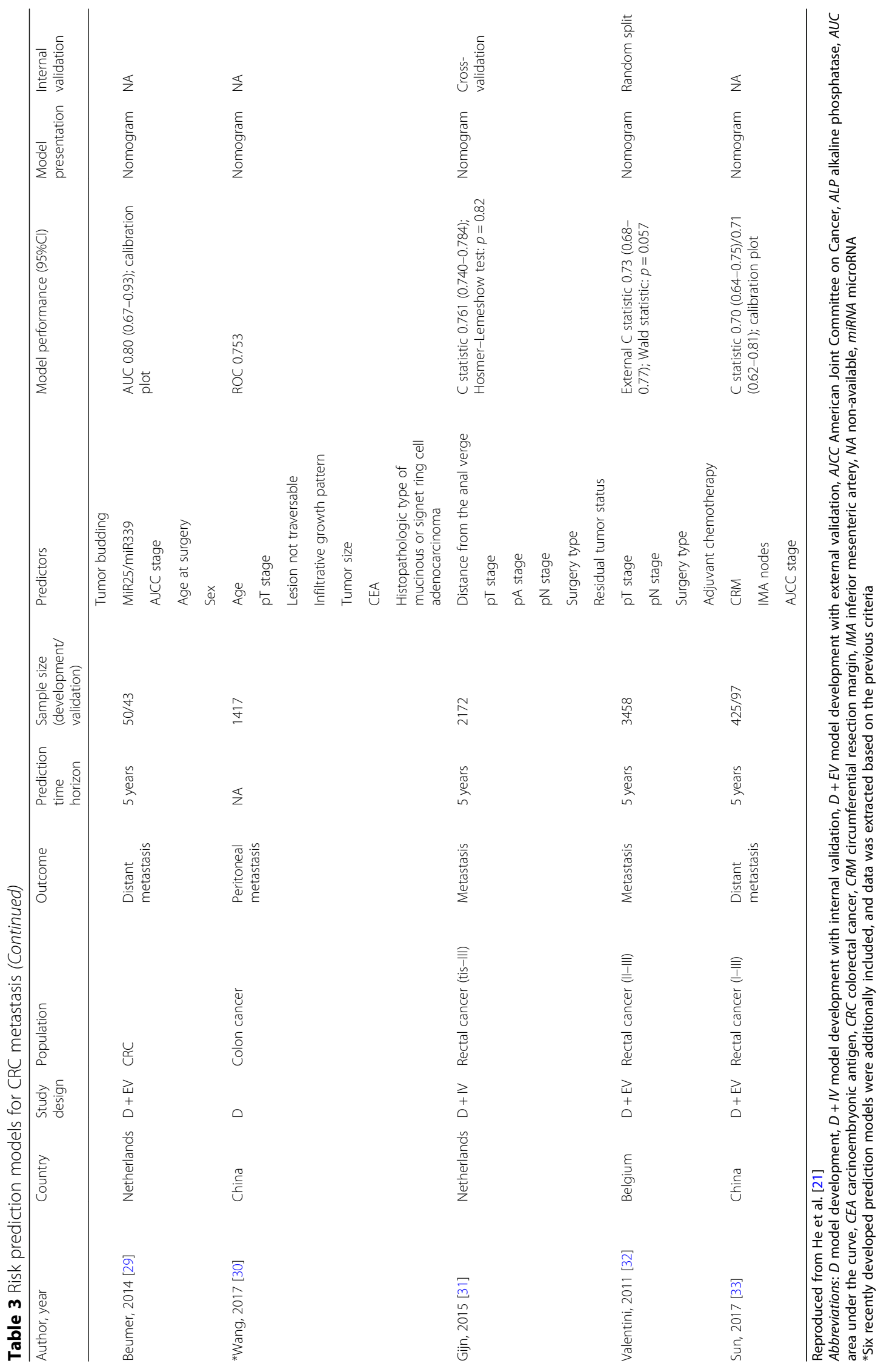


Table 4 Risk prediction models for CRC recurrence

\begin{tabular}{|c|c|c|c|c|c|c|c|c|c|c|}
\hline $\begin{array}{l}\text { Author, } \\
\text { year }\end{array}$ & Country & $\begin{array}{l}\text { Study } \\
\text { design }\end{array}$ & Population & Outcome & $\begin{array}{l}\text { Prediction } \\
\text { time } \\
\text { horizon }\end{array}$ & $\begin{array}{l}\text { Sample size } \\
\text { (development/ } \\
\text { validation) }\end{array}$ & Predictors & $\begin{array}{l}\text { Model } \\
\text { performance } \\
(95 \% \mathrm{Cl})\end{array}$ & $\begin{array}{l}\text { Model } \\
\text { presentation }\end{array}$ & $\begin{array}{l}\text { Internal } \\
\text { validation }\end{array}$ \\
\hline \multirow{7}{*}{$\begin{array}{l}\text { Hoshino, } \\
2016 \text { [34] }\end{array}$} & \multirow[t]{7}{*}{ Japan } & \multirow[t]{7}{*}{ D } & \multirow[t]{7}{*}{ CRC (II) } & \multirow{7}{*}{$\begin{array}{l}\text { Overall } \\
\text { recurrence }\end{array}$} & \multirow[t]{7}{*}{5 years } & \multirow[t]{7}{*}{4167} & Sex & \multirow{7}{*}{$\begin{array}{l}\text { C statistic } 0.64 ; \\
\text { calibration plot }\end{array}$} & \multirow[t]{7}{*}{ Nomogram } & \multirow[t]{7}{*}{ NA } \\
\hline & & & & & & & CEA & & & \\
\hline & & & & & & & Tumor location & & & \\
\hline & & & & & & & Tumor depth & & & \\
\hline & & & & & & & $\begin{array}{l}\text { Lymphatic } \\
\text { invasion }\end{array}$ & & & \\
\hline & & & & & & & Venous invasion & & & \\
\hline & & & & & & & $\begin{array}{l}\text { No. of positive } \\
\text { lymph nodes }\end{array}$ & & & \\
\hline \multirow{2}{*}{$\begin{array}{l}\text { Peng, } \\
2010 \text { [37] }\end{array}$} & \multirow[t]{2}{*}{ China } & \multirow[t]{2}{*}{ D } & \multirow[t]{2}{*}{ CRC (II-III) } & \multirow{2}{*}{$\begin{array}{l}\text { Overall } \\
\text { recurrence }\end{array}$} & \multirow[t]{2}{*}{3 years } & \multirow[t]{2}{*}{95} & AJCC stage & \multirow[t]{2}{*}{ AUC 0.75} & \multirow[t]{2}{*}{ Formula } & \multirow[t]{2}{*}{ NA } \\
\hline & & & & & & & Genetic score & & & \\
\hline \multirow{5}{*}{$\begin{array}{l}\text { Ying, } \\
2014 \text { [38] }\end{array}$} & \multirow[t]{5}{*}{ China } & \multirow[t]{5}{*}{ D } & \multirow{5}{*}{$\begin{array}{l}\text { CRC (I-III, curative } \\
\text { resection) }\end{array}$} & Overall & 3 years & 205 & Tumor size & C statistic & Nomogram & NA \\
\hline & & & & & & & $\begin{array}{l}\text { Tumor } \\
\text { differentiation }\end{array}$ & & & \\
\hline & & & & & & & AJCC stage & & & \\
\hline & & & & & & & NLR & & & \\
\hline & & & & & & & Chemotherapy & & & \\
\hline Zakaria, & Japan & D & CRC (liver & Overall & 5 years & 662 & Hepatoduodenal & C statistic 0.61 & Nomogram & NA \\
\hline & & & hepatectomy) & & & & $\begin{array}{l}\text { Lymph node } \\
\text { status }\end{array}$ & $(0.550 .61)$ & & \\
\hline & & & & & & & Transfusions & & & \\
\hline & & & & & & & Primary cancer & & & \\
\hline & & & & & & & $\begin{array}{l}\text { Regional lymph } \\
\text { node }\end{array}$ & & & \\
\hline & & & & & & & $\begin{array}{l}\text { No. of } \\
\text { metastasis }\end{array}$ & & & \\
\hline Tian, & China & D & CRC & Overall & 3 years & 556 & Gene signature & AUC 0.921 & Nomogram & NA \\
\hline & & & & & & & AJCC stage & calibration plot & & \\
\hline & & & & & & & $\begin{array}{l}\text { Tumor } \\
\text { differentiation }\end{array}$ & & & \\
\hline *Kim, & Korea & $D+I V$ & CRC (I) & Overall & 5 years & 1538 & Sex & C statistic 0.71 ; & Nomogram & NA \\
\hline & & & & & & & Tumor location & & & \\
\hline & & & & & & & pT stage & & & \\
\hline & & & & & & & LVI & & & \\
\hline & & & & & & & Tumor size & & & \\
\hline $\begin{array}{l}{ }^{*} \text { Miyoshi, } \\
2016[41]\end{array}$ & Japan & $\mathrm{D}+\mathrm{EV}$ & $\begin{array}{l}\text { CRC (IV with liver } \\
\text { and/or lung }\end{array}$ & $\begin{array}{l}\text { Overall } \\
\text { recurrence }\end{array}$ & 5 years & 113 & $\begin{array}{l}\text { Preoperative } \\
\text { CEA }\end{array}$ & C statistic 0.631 & Nomogram & NA \\
\hline & & & Metastases) & & & & Tumor location & & & \\
\hline & & & & & & & Tumor invasion & & & \\
\hline & & & & & & & $\begin{array}{l}\text { Lymph node } \\
\text { metastasis }\end{array}$ & & & \\
\hline & & & & & & & $\begin{array}{l}\text { Synchronous } \\
\text { metastatic } \\
\text { lesions }\end{array}$ & & & \\
\hline *Saso, & Japan & $\mathrm{D}+\mathrm{EV}$ & Colon cancer (II) & Overall & 5 years & $352 / 213$ & CEA level & C statistic 0.675 & Nomogram & NA \\
\hline $2010[42]$ & & & & recuntence & & & Tumor invasion & statistic 0.552 & & \\
\hline & & & & & & & $\begin{array}{l}\text { Lymphatic } \\
\text { invasion }\end{array}$ & & & \\
\hline & & & & & & & Venous invasion & & & \\
\hline $\begin{array}{l}\text { Renfro, } \\
2014 \text { [35] }\end{array}$ & USA & $\mathrm{D}+\mathrm{EV}$ & Colon cancer (III) & $\begin{array}{l}\text { Overall } \\
\text { recurrence }\end{array}$ & 5 years & $15,995 / 1903$ & Sex & $\begin{array}{l}\text { C statistic } 0.65 \\
\text { calibration plot }\end{array}$ & Nomogram & NA \\
\hline
\end{tabular}


Table 4 Risk prediction models for CRC recurrence (Continued)

\begin{tabular}{|c|c|c|c|c|c|c|c|c|c|c|}
\hline $\begin{array}{l}\text { Author, } \\
\text { year }\end{array}$ & Country & $\begin{array}{l}\text { Study } \\
\text { design }\end{array}$ & Population & Outcome & $\begin{array}{l}\text { Prediction } \\
\text { time } \\
\text { horizon }\end{array}$ & $\begin{array}{l}\text { Sample size } \\
\text { (development/ } \\
\text { validation) }\end{array}$ & Predictors & $\begin{array}{l}\text { Model } \\
\text { performance } \\
(95 \% \mathrm{Cl})\end{array}$ & $\begin{array}{l}\text { Model } \\
\text { presentation }\end{array}$ & $\begin{array}{l}\text { Internal } \\
\text { validation }\end{array}$ \\
\hline & & & & & & & PS & & & \\
\hline & & & & & & & T stage & & & \\
\hline & & & & & & & $\begin{array}{l}\text { Lymph node } \\
\text { ratio }\end{array}$ & & & \\
\hline & & & & & & & Grade & & & \\
\hline & & & & & & & Tumor location & & & \\
\hline & & & & & & & Treatment & & & \\
\hline \multirow[t]{7}{*}{$\begin{array}{l}\text { Hida, } \\
2017 \text { [43] }\end{array}$} & Japan & D & $\begin{array}{l}\text { Rectal cancer } \\
(I|-| I I)\end{array}$ & $\begin{array}{l}\text { Overall } \\
\text { recurrence }\end{array}$ & 2 years & 792 & $\begin{array}{l}\text { Tumor } \\
\text { differentiation }\end{array}$ & AUC 0.831 & Formula & NA \\
\hline & & & & & & & Depth & & & \\
\hline & & & & & & & Lymph node & & & \\
\hline & & & & & & & Surgery & & & \\
\hline & & & & & & & $\begin{array}{l}\text { Postoperative } \\
\text { complication }\end{array}$ & & & \\
\hline & & & & & & & Tumor height & & & \\
\hline & & & & & & & CEA & & & \\
\hline \multirow[t]{7}{*}{$\begin{array}{l}\text { Gijn, } \\
2015 \text { [31] }\end{array}$} & Netherlands & $D+I V$ & $\begin{array}{l}\text { Rectal cancer } \\
\text { (tis-III) }\end{array}$ & $\begin{array}{l}\text { Local } \\
\text { recurrence }\end{array}$ & 6 years & 1823 & $\begin{array}{l}\text { Distance from } \\
\text { the anal verge }\end{array}$ & $\begin{array}{l}\text { C statistic } 0.787 \\
(0.761-0.814) ;\end{array}$ & Nomogram & Cross-validation \\
\hline & & & & & & & pT stage & $\begin{array}{l}\text { Hosmer-Lemeshow } \\
\text { test: } p=0.68\end{array}$ & & \\
\hline & & & & & & & pN stage & & & \\
\hline & & & & & & & pM stage & & & \\
\hline & & & & & & & Surgery type & & & \\
\hline & & & & & & & $\begin{array}{l}\text { Residual tumor } \\
\text { status }\end{array}$ & & & \\
\hline & & & & & & & Radiotherapy & & & \\
\hline \multirow{6}{*}{$\begin{array}{l}\text { Valentini, } \\
2011[32]\end{array}$} & Belgium & $\mathrm{D}+\mathrm{EV}$ & Rectal cancer & Local & 5 years & 3458 & pT stage & External C statistic & Nomogram & Random split \\
\hline & & & & recurrence & & & cT stage & Wald statistic: $p=0.064$ & & \\
\hline & & & & & & & pN stage & & & \\
\hline & & & & & & & Age & & & \\
\hline & & & & & & & $\begin{array}{l}\text { Concomitant } \\
\text { chemotherapy }\end{array}$ & & & \\
\hline & & & & & & & $\begin{array}{l}\text { Adjuvant } \\
\text { chemotherapy }\end{array}$ & & & \\
\hline
\end{tabular}

Reproduced from He et al. [21]

Abbreviations: $D$ model development, $D+I V$ model development with internal validation, $D+E V$ model development with external validation, $A J C C$ American Joint Committee on Cancer, AUC area under the curve, BMI body mass index, CEA carcinoembryonic antigen, CRC colorectal cancer, LVI lymph vascular invasion, NA non-available, NLR neutrophil to lymphocyte ratio, PS performance status

*Three recently developed prediction models were additionally included, and data was extracted based on the previous criteria

models. Other common predictor variables included histopathological risk factors such as positive lymph nodes $(N=5,42 \%)$, tumor grade or differentiation $(N=$ $4,33 \%)$, and tumor size $(N=4,33 \%)$; biomarker-CEA $(N=4,33 \%)$; cancer location $(N=4,33 \%)$; and clinical treatment such as surgery, chemotherapy, radiotherapy, and transfusion $(N=5,42 \%)$. Two models (17\%) performed internal validation, and 4 models (33\%) were validated in external datasets.

In our cross-evaluation, five of 25 unique predictors (intramural vascular invasion, extramural vascular invasion, being underweight, being overweight, and being obese) were evaluated in the umbrella review (Table 5). The associated ORs for these five factors varied from 1.00 to 3.91 , and only one (20\%) (extramural vascular invasion) corresponded to $\geq 3$-fold change in the odds of the outcome. Of the remaining 12 factors evaluated in the umbrella review, ORs varied from 0.07 to 5.50, and three $(25 \%)$ represented $\geq 3$-fold change in the odds of the outcome.

In relation to overlapping outcomes, only one outcome (overall recurrence in CRC) was identified (Table 6). However, the prognostic model [36] included risk predictors that were not evaluated in the umbrella review 
Table 5 Cross-assessment of the same risk factors and risk predictors

\begin{tabular}{|c|c|c|c|c|c|c|c|}
\hline $\begin{array}{l}\text { Risk factor/risk } \\
\text { predictor }\end{array}$ & $\begin{array}{l}\text { Outcome evaluated } \\
\text { in the umbrella review }\end{array}$ & $\begin{array}{l}\text { Risk factor } \\
\text { prevalence }\end{array}$ & $\begin{array}{l}\text { Effect size } \\
(95 \% \mathrm{Cl})^{\mathbf{a}}\end{array}$ & $\begin{array}{l}\text { Credibility } \\
\text { assessment }\end{array}$ & $\begin{array}{l}\text { Outcome in the risk } \\
\text { prediction models }\end{array}$ & $\begin{array}{l}\text { Effect size } \\
(95 \% \mathrm{Cl})^{\mathbf{b}}\end{array}$ & $\begin{array}{l}\text { Model } \\
\text { performance }\end{array}$ \\
\hline \multicolumn{8}{|l|}{ CRC metastasis } \\
\hline \multicolumn{8}{|c|}{ Histopathological risk factor } \\
\hline \multirow[t]{3}{*}{$\begin{array}{l}\text { Vascular } \\
\text { invasion }\end{array}$} & $\begin{array}{l}\text { Lymph node metastasis } \\
\text { in pT1 CRC }\end{array}$ & $330 / 1731=19 \%$ & $2.73(1.98-3.78)$ & Convincing & \multirow{3}{*}{$\begin{array}{l}\text { Lymph node } \\
\text { metastasis in } \\
\text { pT1 CRC }\end{array}$} & \multirow[t]{3}{*}{$8.45(4.56-15.66)$} & \multirow{3}{*}{$\begin{array}{l}\text { AUC } 0.812(0.770-0.855) \text {; } \\
\text { Hosmer-Lemeshow test: } \\
p=0.737 \text { (55) }\end{array}$} \\
\hline & $\begin{array}{l}\text { Lymph node metastasis } \\
\text { in rectal cancer }\end{array}$ & $46 / 168=27 \%$ & $5.86(0.77-44.62)$ & $\begin{array}{l}\text { No } \\
\text { association }\end{array}$ & & & \\
\hline & $\begin{array}{l}\text { Lymph node metastasis } \\
\text { in small rectal NETs } \\
\text { treated by local excision }\end{array}$ & $75 / 211=36 \%$ & $3.63(0.05-268.57)$ & $\begin{array}{l}\text { No } \\
\text { association }\end{array}$ & & & \\
\hline \multirow[t]{2}{*}{$\begin{array}{l}\text { Tumor } \\
\text { budding }\end{array}$} & $\begin{array}{l}\text { Lymph node metastasis } \\
\text { in pT1 CRC }\end{array}$ & $2401 / 10,128=24 \%$ & $6.39(5.23-7.80)$ & $\begin{array}{l}\text { Highly } \\
\text { suggestive }\end{array}$ & \multirow{2}{*}{$\begin{array}{l}\text { Lymph node } \\
\text { metastasis in } \\
\text { pT1 CRC }\end{array}$} & \multirow[t]{2}{*}{$1.70(1.03-2.80)$} & \multirow{2}{*}{$\begin{array}{l}\text { AUC } 0.812(0.770-0.855) \text {; } \\
\text { Hosmer-Lemeshow test: } \\
p=0.737 \text { (55) }\end{array}$} \\
\hline & $\begin{array}{l}\text { Lymph node metastasis } \\
\text { in CRC }\end{array}$ & $1955 / 6739=29 \%$ & $4.96(3.97-6.19)$ & $\begin{array}{l}\text { Highly } \\
\text { suggestive }\end{array}$ & & & \\
\hline \multirow[t]{2}{*}{$\begin{array}{l}\text { Tumor } \\
\text { differentiation }\end{array}$} & $\begin{array}{l}\text { Lymph node metastasis } \\
\text { in pT1 CRC }\end{array}$ & $94 / 2722=4 \%$ & $5.61(2.90-10.83)$ & Suggestive & $\begin{array}{l}\text { Lymph node } \\
\text { metastasis in } \\
\text { pT1 CRC }\end{array}$ & $11.77(0.77-179.83)$ & $\begin{array}{l}\text { AUC } 0.90 \\
(0.81-0.99)(49)\end{array}$ \\
\hline & $\begin{array}{l}\text { Lymph node metastasis } \\
\text { in pT1 CRC patients who } \\
\text { underwent additional } \\
\text { surgeries after an } \\
\text { endoscopic resection }\end{array}$ & $122 / 209=58 \%$ & $3.77(1.12-123.16)$ & $\begin{array}{l}\text { No } \\
\text { association }\end{array}$ & $\begin{array}{l}\text { Synchronous } \\
\text { bone metastasis }\end{array}$ & $1.69(1.22-2.32)$ & $\begin{array}{l}\text { AUC } 0.903 \text {; sensitivity } \\
0.851 \text {; specificity } \\
0.845 \text { (54) }\end{array}$ \\
\hline $\begin{array}{l}\text { Submucosal } \\
\text { invasion } \geq 1 \\
\mathrm{~mm}\end{array}$ & $\begin{array}{l}\text { Lymph node metastasis } \\
\text { in pT1 CRC }\end{array}$ & $2389 / 2922=82 \%$ & $2.95(1.39-6.27)$ & Weak & $\begin{array}{l}\text { Lymph node } \\
\text { metastasis in } \\
\text { pT1 CRC }\end{array}$ & $2.14(1.19-3.86)$ & $\begin{array}{l}\text { AUC } 0.812(0.770-0.855) \\
\text { Hosmer-Lemeshow test: } \\
p=0.737 \text { (55) }\end{array}$ \\
\hline $\begin{array}{l}\text { Tumor size > } \\
1 \mathrm{~cm}\end{array}$ & $\begin{array}{l}\text { Lymph node metastasis } \\
\text { in rectal cancer }\end{array}$ & $203 / 348=58 \%$ & $6.76(3.25-14.04)$ & $\begin{array}{l}\text { Highly } \\
\text { suggestive }\end{array}$ & $\begin{array}{l}\text { Peritoneal } \\
\text { metastasis in } \\
\text { colon cancer }\end{array}$ & $1.04(1.00-1.09)$ & ROC $0.753(57)$ \\
\hline \multicolumn{8}{|c|}{ Demographic risk factor } \\
\hline \multirow[t]{2}{*}{ Sex/gender } & \multirow[t]{2}{*}{$\begin{array}{l}\text { Lymph node metastasis } \\
\text { in pT1 CRC }\end{array}$} & \multirow[t]{2}{*}{$465 / 1329=35 \%$} & \multirow[t]{2}{*}{$2.23(0.78-6.42)$} & \multirow[t]{2}{*}{$\begin{array}{l}\text { No } \\
\text { association }\end{array}$} & $\begin{array}{l}\text { Liver metastasis } \\
\text { in CRC }\end{array}$ & NA & $\begin{array}{l}\text { Sensitivity } 0.74 ; \\
\text { specificity } 0.62(50)\end{array}$ \\
\hline & & & & & $\begin{array}{l}\text { Distant metastasis } \\
\text { in CRC }\end{array}$ & $1.40(0.46-4.28)$ & $\begin{array}{l}\text { AUC } 0.80(0.67-0.93) \\
\text { calibration plot }(56)\end{array}$ \\
\hline \multicolumn{8}{|l|}{ CRC recurrence } \\
\hline \multicolumn{8}{|c|}{ Histopathological risk factor } \\
\hline $\begin{array}{l}\text { Vascular } \\
\text { invasion } \\
\text { (intramural) }\end{array}$ & Local recurrence in CRC & $137 / 503=27 \%$ & $1.55(0.11-21.28)$ & $\begin{array}{l}\text { No } \\
\text { association }\end{array}$ & $\begin{array}{l}\text { Overall recurrence } \\
\text { in stage II CRC }\end{array}$ & $1.30(1.07-1.58)$ & $\begin{array}{l}\text { C statistic } 0.64 ; \\
\text { calibration plot }(61)\end{array}$ \\
\hline $\begin{array}{l}\text { Vascular } \\
\text { invasion } \\
\text { (extramural) }\end{array}$ & $\begin{array}{l}\text { Distant metastatic } \\
\text { recurrence in rectal } \\
\text { cancer }\end{array}$ & $350 / 1262=28 \%$ & $3.91(2.61-5.86)$ & $\begin{array}{l}\text { Highly } \\
\text { suggestive }\end{array}$ & $\begin{array}{l}\text { Overall recurrence } \\
\text { in stage II colon } \\
\text { cancer }\end{array}$ & $2.48(1.22-5.57)$ & $\begin{array}{l}\text { C statistic } 0.675 ; \\
\text { external C statistic } \\
0.552(68)\end{array}$ \\
\hline \multicolumn{8}{|c|}{ Anthropometric indices } \\
\hline $\begin{array}{l}\text { BMI } \\
\text { (underweight) }\end{array}$ & $\begin{array}{l}\text { Overall recurrence in } \\
\text { CRC }\end{array}$ & $2752 / 17,636=16 \%$ & $1.13(1.05-1.21)$ & Weak & \multirow{3}{*}{$\begin{array}{l}\text { Overall recurrence } \\
\text { in stage III colon } \\
\text { cancer }\end{array}$} & \multirow[t]{3}{*}{ NA } & \multirow[t]{3}{*}{$\begin{array}{l}\text { C statistic } 0.65 \\
\text { calibration plot (69) }\end{array}$} \\
\hline $\begin{array}{l}\text { BMI } \\
\text { (overweight) }\end{array}$ & $\begin{array}{l}\text { Overall recurrence in } \\
\text { CRC }\end{array}$ & $13,225 / 28,109=47 \%$ & $1.00(0.96-1.05)$ & $\begin{array}{l}\text { No } \\
\text { association }\end{array}$ & & & \\
\hline BMI (obese) & $\begin{array}{l}\text { Overall recurrence in } \\
\text { CRC }\end{array}$ & $6362 / 21,246=30 \%$ & $1.07(1.02-1.13)$ & Weak & & & \\
\hline
\end{tabular}

Abbreviations: $A U C$ area under the curve, $B M I$ body mass index, $C l$ confidence interval, $C R C$ colorectal cancer, $N A$ non-available

${ }^{a}$ Effect size $(95 \% \mathrm{Cl})$, effect size from the umbrella review

${ }^{\mathrm{b}}$ Effect size $(95 \% \mathrm{Cl})$, effect size from the risk prediction models

(cancer stage, tumor differentiation, and gene signature). Meanwhile, within the evaluated nine risk factors for overall recurrence in CRC that were not employed as predictors in this model, only two influential risk factors (tumor budding, absence of peritoneal free tumor cells in post-resection) had $\geq 3$-fold change in the odds of the outcome.

\section{Discussion}

We initially synthesized and evaluated the evidence of risk factors for CRC metastasis and recurrence. Our study comprised 51 unique meta-analyses of observational studies investigating 34 risk factors for CRC metastasis and 17 risk factors for recurrence. We also conducted a sensitivity analysis of 29 unique meta- 


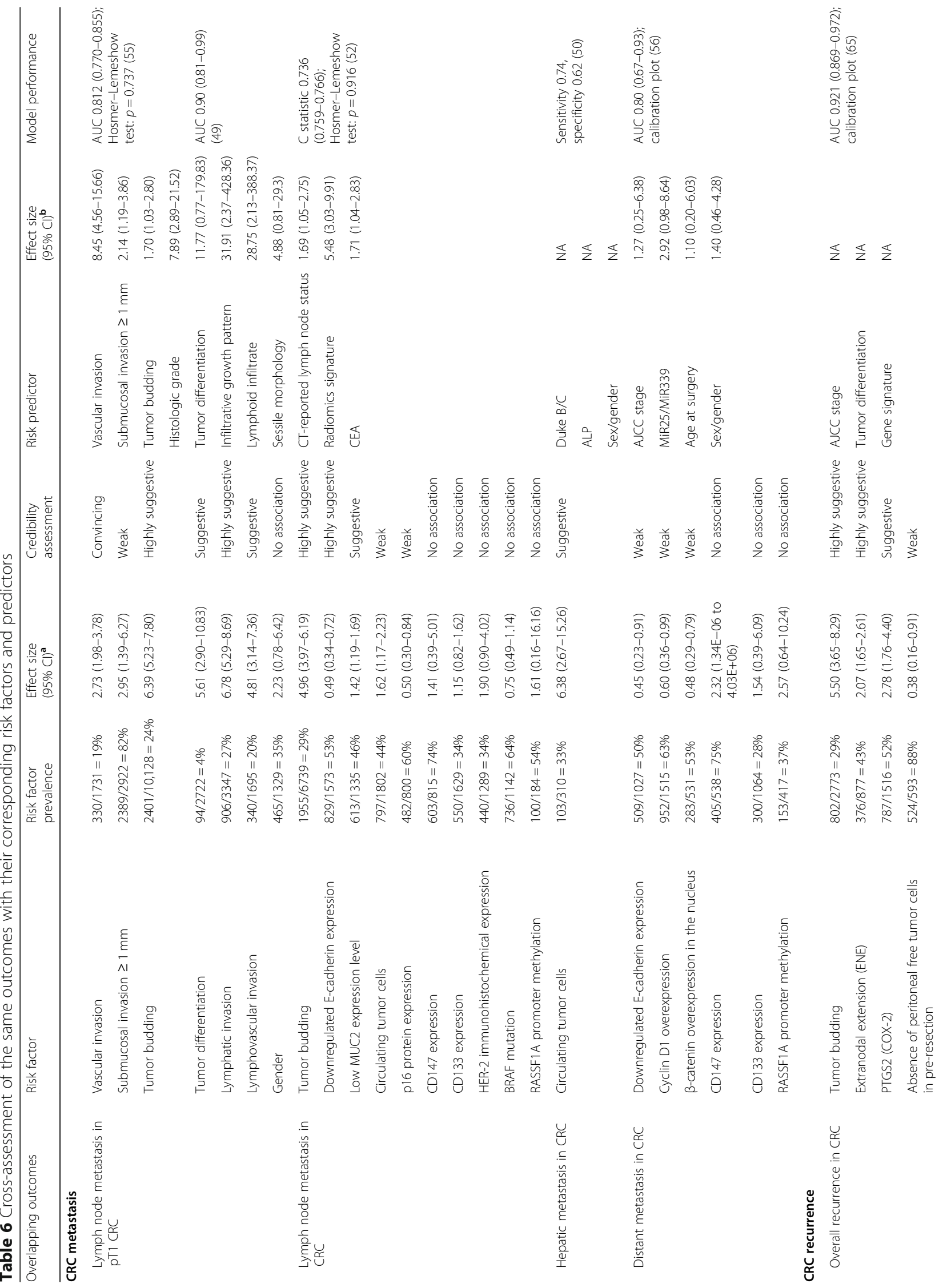




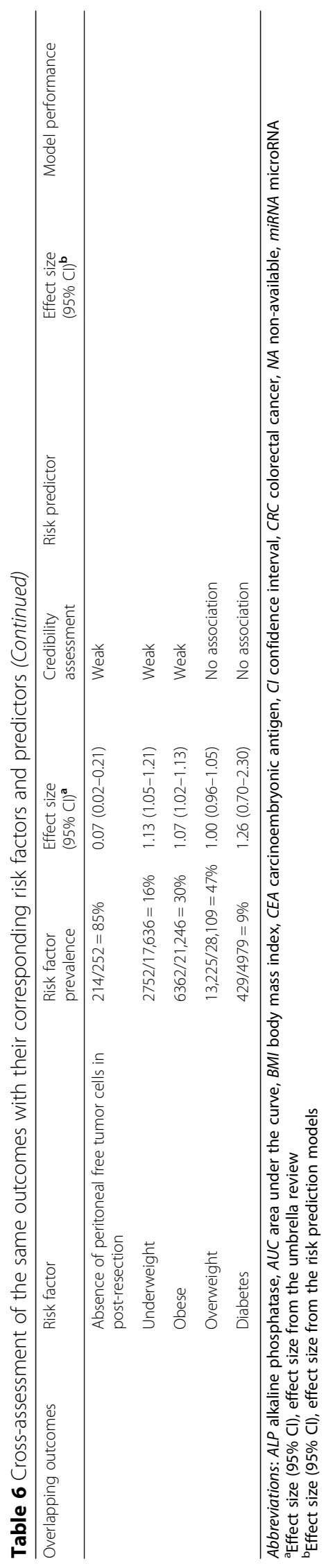


analyses of risk factors for CRC metastasis at presentation $(n=16)$, CRC local recurrence $(n=5)$, and CRC distant recurrence $(n=8)$ using a standardized categorization of the component studies. Furthermore, we updated synthesis of risk prediction models for CRC metastasis $(n=12)$ and recurrence $(n=12)$ and then conducted a cross-assessment of individual risk factors evaluated in the umbrella review and risk predictors included in existing prediction models, which allowed us to examine to what extent predictive models include the most influential factors.

\section{Main findings and interpretation of the umbrella review Meta-analyses for CRC metastasis}

According to our pre-defined criteria for assessing the credibility of the evidence, only one risk factor was classified as convincing (vascular invasion for LNM in pT1 $C R C$ ), reflecting strong statistical significance and no hints of bias. Many studies have demonstrated that the invasion of blood vessels leading to tumor cell dissemination and metastasis is a strong risk factor for disease prognosis, which is in line with our umbrella review [44, 45]. Based on our findings, a large proportion of studies $(17 / 25,68 \%)$ investigated lymphatic and vascular invasion as separate risk factors, while $32 \%$ of studies categorized them jointly as lymphovascular invasion. It has been shown though that the predictive ability of lymphovascular invasion is lower than that of vascular invasion [46].

Twelve (35\%) of 34 probed risk factors for metastasis had an effect size suggesting $\geq 3$-fold change in the odds of the outcome with $p<0.05$. Four of these risk factors (lymphatic invasion for LNM in pT1 CRC; tumor budding for LNM in pT1 CRC; tumor budding for LNM in all stage CRC; tumor size $>1 \mathrm{~cm}$ for LNM in rectal cancer) were classified as highly suggestive. As discussed above, lymphatic invasion could be an indicator of tumor cells metastasizing to lymph nodes. This finding agrees with three recently published studies manifesting that lymphatic invasion is causally associated with the risk of LNM in CRC [47-49]. Tumor budding is recognized as a negative prognostic risk factor for LNM in $\mathrm{CRC}$, and our findings are concordant with previous studies [50-52]. Individual component studies vary in their definitions of tumor budding (e.g., how many cancer cells comprise a tumor bud, and how many buds signify tumor budding) and vary in the pathologic staining methods to detect tumor budding (e.g., hematoxylin and eosin $[H \& E]$, immunohistochemistry [IHC]). Furthermore, a systematic review summarized pathologic methods to detect tumor budding and revealed that all studies even when utilizing different methods showed that tumor budding increases the risk of CRC metastasis [53]. Notably, substantial between-study heterogeneity
$\left(I^{2}>50 \%\right)$ was found in the meta-analysis investigating tumor budding for LNM in all CRC stages, indicating that this association needs to be interpreted with caution. The observed heterogeneity may be influenced by the inclusion of different tumor stages. Finally, tumor size $>1 \mathrm{~cm}$ is associated with an increased risk of LNM in rectal cancer. This largely agrees with the European Society for Medical Oncology (ESMO) clinical practice guideline manifesting that a rectal lesion less than $1 \mathrm{~cm}$ has a lower risk of metastasis, and therefore, local excision (TEM) is suggested [54].

\section{Meta-analyses for CRC recurrence}

In regard to 17 probed risk factors for CRC recurrence, four (24\%) had an effect size suggesting $\geq 3$-fold change in the odds of the outcome with $p<0.05$. None of them presented convincing evidence. Three (tumor budding for overall recurrence in CRC; perineural invasion [PNI] for local recurrence in rectal cancer; MRI-detected extramural vascular invasion [mrEMVI] for distant metastatic recurrence in rectal cancer) were classified as highly suggestive. Our findings suggest that tumor budding is a common highly suggestive risk factor for both CRC LNM and overall recurrence. However, there is a need for standardization of the histopathological definition of tumor budding [46]. Another histopathological risk factor, $P N I$, which is a common pathological feature in rectal cancer, strongly signifies local recurrence. Compared to colon cancer, PNI occurs more frequently in rectal cancer, since there is a cluster of intensive neural plexuses surrounding the pelvis in the rectum [55]. The National Comprehensive Cancer Network (NCCN) guidelines also suggest that patients with PNI positive are at higher risk of local recurrence [56]. However, there is no consensus in the definition of PNI positive, with two of the most frequently used definitions being SS-PNI (when tumor cells surround at least 33\% of the nerve) and TS-PNI (when tumor cells surround any of the three layers of the nerve) [57-60]. Finally, we found that $m r E M V I$ increases the risk of distant metastatic recurrence. EMVI is the venous invasion beyond the muscularis propria, which has long been recognized as a risk factor for distant recurrence [61-63]. The 5-point MRIdetected EMVI scoring system is precise for detecting this invasion, and it is recommended as a post-operation follow-up strategy in clinical settings [64]. In addition, a recently published meta-analysis is also in line with our findings, reporting that around $90 \%$ of patients with liver metastases are mrEMVI positive [65].

\section{Sensitivity analysis}

In our effort for a consistent definition of metastasis and recurrence, we re-categorized all the component studies to three distinct disease outcomes: metastasis at 
presentation, local recurrence, and distant recurrence. This could generate insight into metastasis and recurrence patterns and provide investigators and clinicians with a more comprehensive summary of risk factors for these CRC prognostic outcomes with clinical significance [66]. Our sensitivity analyses reported a dearth of convincing evidence. However, a total of 244 (62\%) individual component studies were excluded from our sensitivity analyses due to missing information in relation to outcome definition.

\section{Cross-assessment between risk factors evaluated in the umbrella review and risk predictors applied in existing prediction models}

We identified 24 CRC prognostic models for metastasis $(n=12)$ and recurrence $(n=12)$. The majority of risk prediction models applied an average of four to five predictor variables. The most commonly used predictors were clinic-histopathological (cancer stage, lymph node status) and demographic (gender, age) parameters. Seven models were validated internally and eight in external datasets, but none of the identified models conducted any impact studies. As for model presentation, the majority of models were nomograms (graphical prediction models), and the remaining models were presented as formulae, risk scores, and calculators.

In our cross-assessment, we investigated whether the identified prediction models had employed influential risk factors (those presented convincing evidence or with 3 -fold change in the odds of the outcome) when they predicted the same outcomes as those that were evaluated in the umbrella review. Across 12 CRC metastasis risk prediction models, five models $[22,23,25,28,29]$ were on the same outcomes (LNM in pT1 CRC, LNM in CRC, hepatic metastasis in CRC, and distant metastasis in CRC), with only two $[22,28]$ of these models (on LNM in pT1 $C R C$ ) including predictors also evaluated in the umbrella review. However, the models' calibration was poorly reported, which made it difficult to assess the models' predictive accuracy. Furthermore, one model [28] was externally validated to ensure the model's applicability and generalizability, while the remaining one [22] did not undergo adequate validation to address its potential overfitting. In addition, the remaining three models $[23,25,29]$ predicting LNM and DM in CRC applied other risk predictors such as cancer stage, CEA, and alkaline phosphatase (ALP) that were not evaluated in the umbrella review. We suggest that risk factors with strong associations with CRC prognosis, such as circulating tumor cells and microsatellite instability, should be employed following evidence-based methods.
Across the 12 CRC recurrence risk prediction models, only one model [36] was on an outcome that was also evaluated in the umbrella review (overall recurrence in CRC). Unfortunately, we did not find overlapping risk factors/ predictors. We recommend tumor budding and absence of peritoneal free tumor cells in post-resection $(\geq 3$-fold change in the odds of the outcome) to be considered as predictors.

\section{Clinical implications and future research}

Identifying and evaluating risk factors with substantial predictive value is of great clinical importance. Major clinical decisions are made taking into account expectations and formal or informal predictions about major outcomes. Accurate and valid risk prediction could assist with clinical decision-making in relation to the extent and mode of surgery and therapy. Ideally, adjuvant treatment would be targeted with precision to those most likely to benefit; those most at risk of CRC metastasis/ recurrence may also have a higher absolute probability of benefit. The majority of patients do not benefit from additional therapy aimed at preventing locoregional or distant relapse before or after surgical resection, and yet they may be exposed to the attendant morbidity, cost, and false expectation of such therapy. Therefore, accurate and valid risk prediction which could impact clinical decision-making is crucial. In summary, this umbrella review provides an evidence classification that could help clinicians to judge the relative priority of risk factors/predictors' impact on CRC prognosis and make clinical decisions based on more accurate and valid risk prediction.

Our findings suggest that efforts to address the limitations of the available evidence could be beneficial. Largescale prospective studies are needed to generate evidence less prone to bias and allowing better predictive model building and validation. Standardizing the outcome definitions of CRC metastasis and recurrence could improve reporting of outcomes that have direct clinical relevance. Future risk prediction model research is encouraged to apply rigorous model construction processes and to integrate the most influential risk factors based on evidencebased methods.

\section{Strengths and limitations}

The main strength of this study is that it provides a rigorous critical assessment of the published epidemiological evidence on risk factors of CRC metastasis and recurrence, based on pre-defined criteria in a transparent and systematic way $[17,18]$. In addition, we updated the synthesis of CRC prognostic prediction models, and to our best knowledge, this is the first cross-assessment between individual risk factors and risk predictors applied in existing prediction models, to investigate whether influential risk factors are employed as 
predictors. Our findings provide a comprehensive evaluation of available evidence that can inform future research on risk factors for CRC prognostic outcomes and risk prediction models.

However, the following potential limitations should be considered. First, umbrella review comprises a synthesis of evidence from existing systematic reviews and metaanalyses [67]. Therefore, risk factors and risk predictors that were not systematically reviewed in the pre-existing literature are not included in this umbrella review. These may include some factors that are commonly used in predictive models, and it highlights the need to perform systematic reviews of the evidence for factors that might be routinely or frequently measured. Second, metaanalyses have common defects such as limited coverage of the literature search and low quality of the included studies [68, 69]. Third, this study only collected and evaluated evidence from systematic reviews and metaanalyses of observational studies published in peerreviewed journals. This could limit the breadth of our results if research in gray literature, conference abstracts, and comments investigated risk factors that were not included in this umbrella review. Furthermore, $77 \%$ of meta-analyses included only retrospective studies.

Moreover, this study did not evaluate the quality of all individual component studies included in each metaanalysis because it is beyond the scope of an umbrella review. Instead, we performed a credibility evaluation and risk of bias assessment for meta-analyses that represented at least 3-fold changes in the odds of the outcome. Criteria for assessing the evidence from metaanalyses of observational studies applied in our umbrella review were based on pre-defined metrics whose limitations have been summarized [70-72]. For the outcomes that we studied, one is probably interested usually on whether the considered risk factors confer substantial predictive value, rather than whether they are causally related to the outcomes. We pre-specified a threshold for the magnitude of what might be a relatively large effect size (3-fold change in odds), but this is not absolute. The predictive value may depend also on how frequently a given factor is in the evaluated population. However, with one exception, all the factors evaluated concurrently in both risk factor meta-analyses and in predictive models were pretty common, with prevalence ranging from 16 to $82 \%$.

We should also acknowledge that although we performed a sensitivity analysis to classify CRC metastasis at presentation, local or distant recurrence, a large proportion $(62 \%)$ of individual component studies did not present enough information, such as the timing of metastasis in relation to initial diagnosis (i.e., synchronous or metachronous) and local or distant recurrence separately from overall recurrence. Finally, we did not evaluate risk factors relevant to clinical interventions such as surgery type, chemotherapy, radiotherapy, and transfusion. We also could not perform a complete comparison between risk factors evaluated in the umbrella review and risk predictors applied in existing prediction models because only 11 overlapping risk factors/predictors were identified.

\section{Conclusions}

In this umbrella review, we synthesized and evaluated risk factors and risk prediction models of CRC metastasis and recurrence. A total of 51 unique risk factors were investigated, convincing evidence exists only for the association between vascular invasion and LNM, and even that is restricted to pT1 tumors. Furthermore, we also conducted a cross-assessment to evaluate individual risk factors and risk prediction models. Our findings emphasize the need for a more rigorous and systematic model construction process to integrate influential risk factors following evidence-based methods.

\section{Supplementary information}

Supplementary information accompanies this paper at https://doi.org/10. 1186/s12916-020-01618-6.

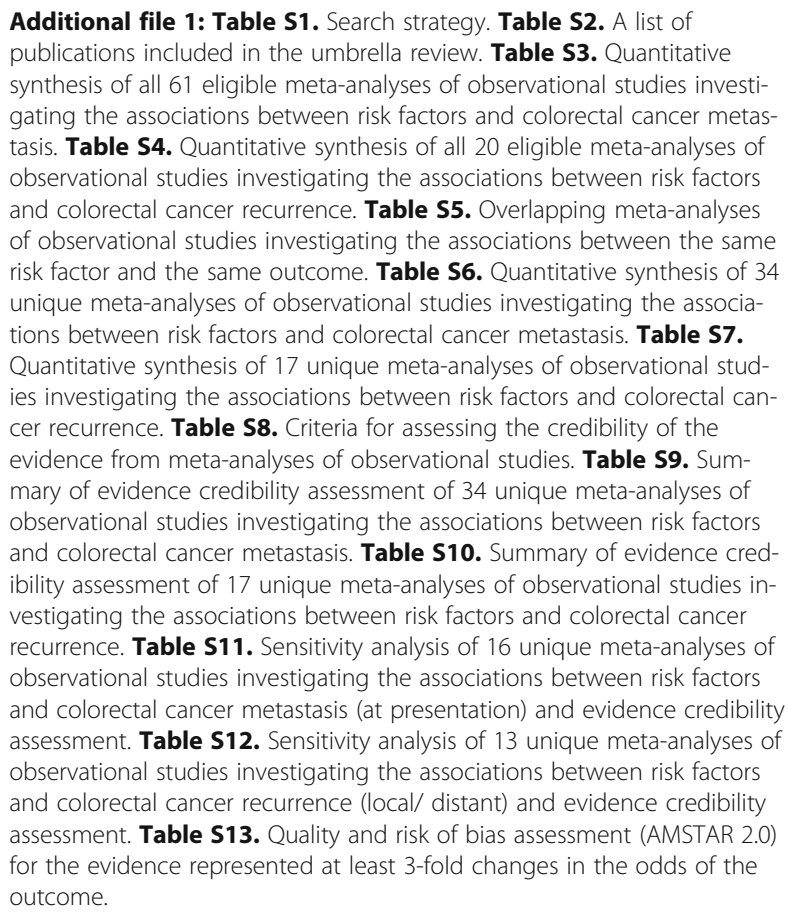

\section{Abbreviations}

AMSTAR 2.0: Assessment of Multiple Systematic Reviews 2.0; ALP: Alkaline phosphatase; CEA: Carcinoembryonic antigen; Cl: Confidence interval; CRC: Colorectal cancer; DL: DerSimonian and Laird; EMR: Endoscopic resection; ESMO: European Society for Medical Oncology; H\&E: Hematoxylin and eosin; HKSJ: Hartung-Knapp-Sidik-Jonkman; HR: Hazard ratio; IHC: Immunohistochemistry; LNM: Lymph node metastasis; mrEMVI: MRIdetected extramural vascular invasion; NCCN: National Comprehensive 
Cancer Network; OR: Odds ratio; PNI: Perineural invasion; PRISMA-P: Preferred Reporting Items for Systematic Reviews and Meta-Analyses Protocols; RR: Risk ratio; TEM: Trans-anal microscopic surgery

\section{Acknowledgements}

Not applicable.

\section{Authors' contributions}

E. Theodoratou., M.G. Dunlop, and J.P.A. loannidis designed the study. W. Xu and $Y$. Wang performed the literature search, the selection of eligible articles, and the data extraction. W. Xu analyzed the data, and Y. He checked the data. W. Xu and E. Theodoratou wrote the first draft of the manuscript. All authors were involved in the revision of the manuscript and approved the final version of the submitted manuscript.

\section{Funding}

The work was supported by a Programme Grant from Cancer Research UK C348/A18927. It was also supported by funding for the infrastructure and staffing of the Edinburgh CRUK Cancer Research Centre. This work was also supported by a grant to M.G. Dunlop as Project Leader with the MRC Human Genetics Unit Centre Grant (U127527202 and U127527198 from 1/4/ 18). E. Theodoratou has a Career Development Fellowship from Cancer Research UK [grant number C31250/A22804]

\section{Availability of data and materials \\ Not applicable.}

\section{Ethics approval and consent to participate}

Not applicable.

\section{Consent for publication}

Not applicable.

\section{Competing interests}

The authors declare that they have no competing interests.

\section{Author details}

${ }^{1}$ Centre for Global Health, Usher Institute, University of Edinburgh, Edinburgh EH8 9AG, UK. ²Henan Provincial People's Hospital, Henan 450003, People's Republic of China. ${ }^{3}$ Sydney School of Public Health, University of Sydney, Sydney, NSW 2006, Australia. ${ }^{4}$ Department of Medicine, School of Medicine, Stanford University, Stanford, CA 94305, USA. ${ }^{5}$ Department of Epidemiology and Population Health, School of Medicine, Stanford University, Stanford, CA 94305, USA. 'Department of Biomedical Data Science, School of Medicine, Stanford University, Stanford, CA 94305, USA. ${ }^{7}$ Meta-Research Innovation Center at Stanford (METRICS), Stanford University, Stanford, CA 94305, USA. ${ }^{8}$ Department of Statistics, School of Humanities and Sciences, Stanford University, Stanford, CA 94305, USA. ${ }^{9}$ Colon Cancer Genetics Group, Medical Research Council Human Genetics Unit, Medical Research Council Institute of Genetics \& Molecular Medicine, University of Edinburgh, Edinburgh EH4 2XU, UK. ${ }^{10}$ Edinburgh Cancer Research Centre, Institute of Genetics and Molecular Medicine, University of Edinburgh, Edinburgh EH4 2XU, UK.

\section{Received: 24 February 2020 Accepted: 7 May 2020}

\section{Published online: 26 June 2020}

\section{References}

1. Riihimäki M, Hemminki A, Sundquist J, et al. Patterns of metastasis in colon and rectal cancer. Sci Rep. 2016;6:29765.

2. Mekenkamp LJ, Koopman M, Teerenstra S, et al. Clinicopathological features and outcome in advanced colorectal cancer patients with synchronous vs metachronous metastases. Br J Cancer. 2010;103(2):159.

3. Duineveld LA, van Asselt KM, Bemelman WA, et al. Symptomatic and asymptomatic colon cancer recurrence: a multicenter cohort study. Ann Fam Med. 2016;14(3):215-20.

4. Ryuk JP, Choi GS, Park JS, et al. Predictive factors and the prognosis of recurrence of colorectal cancer within 2 years after curative resection. Ann Surg Treatment Res. 2014;86(3):143-51.

5. Dromain C, Caramella C, Dartigues P, et al. Liver, lung and peritoneal metastases in colorectal cancers: is the patient still curable? What should the radiologist know. Diagnostic Interventional Imaging. 2014;95(5):513-23.
6. Haggar FA, Boushey RP. Colorectal cancer epidemiology: incidence, mortality, survival, and risk factors. Clinics Colon Rectal Surg. 2009;22(04):191-7.

7. Beaton $\mathrm{C}$, Twine $\mathrm{CP}$, Williams $\mathrm{GL}$, et al. Systematic review and meta-analysis of histopathological factors influencing the risk of lymph node metastasis in early colorectal cancer. Color Dis. 2013;15(7):788-97.

8. Choi JY, Jung S, Shim KN, et al. Meta-analysis of predictive clinicopathologic factors for lymph node metastasis in patients with early colorectal carcinoma. J Korean Med Sci. 2015;30(4):398-406.

9. Ha GW, Kim JH, Lee MR. Oncologic impact of anastomotic leakage following colorectal cancer surgery: a systematic review and meta-analysis. Ann Surg Oncol. 2017;24(11):3289-99.

10. Rekhraj S, Aziz O, Prabhudesai S, et al. Can intra-operative intraperitoneal free cancer cell detection techniques identify patients at higher recurrence risk following curative colorectal cancer resection: a meta-analysis. Ann Surg Oncol. 2008;15(1):60-8.

11. Moher D, Shamseer L, Clarke M, et al. Preferred reporting items for systematic review and meta-analysis protocols (PRISMA-P) 2015 statement. Systematic Reviews. 2015;4(1):1.

12. DerSimonian R, Laird N. Meta-analysis in clinical trials. Control Clin Trials. 1986;7(3):177-88.

13. IntHout J, loannidis JP, Borm GF. The Hartung-Knapp-Sidik-Jonkman method for random effects meta-analysis is straightforward and considerably outperforms the standard DerSimonian-Laird method. BMC Med Res Methodol. 2014;14(1):25

14. Higgins JP, Thompson SG, Deeks JJ, et al. Measuring inconsistency in metaanalyses. Bmj. 2003;327(7414):557-60.

15. Egger $M$, Smith GD, Schneider $M$, et al. Bias in meta-analysis detected by a simple, graphical test. Bmj. 1997;315(7109):629-34.

16. loannidis JP. Clarifications on the application and interpretation of the test for excess significance and its extensions. J Math Psychol. 2013; 57(5):184-7.

17. He Y, Li X, Gasevic D, et al. Statins and multiple noncardiovascular outcomes: umbrella review of meta-analyses of observational studies and randomized controlled trials. Ann Intern Med. 2018;169(8):543-53. .

18. Li X, Meng X, Timofeeva M, et al. Serum uric acid levels and multiple health outcomes: umbrella review of evidence from observational studies, randomised controlled trials, and Mendelian randomisation studies. BMJ. 2017;357:j2376.

19. Shea BJ, Reeves BC, Wells G, et al. AMSTAR 2: a critical appraisal tool for systematic reviews that include randomised or non-randomised studies of healthcare interventions, or both. Bmj. 2017;358:j4008.

20. Guyatt $\mathrm{GH}$, Oxman AD, Sultan S, et al. GRADE guidelines: 9. Rating up the quality of evidence. J Clin Epidemiol. 2011;64(12):1311-6.

21. He Y, Ong Y, Li X, et al. Performance of prediction models on survival outcomes of colorectal cancer with surgical resection: a systematic review and meta-analysis. Surg Oncol. 2019;29:196-202.

22. Macias-Garcia F, Celeiro-Muñoz C, Lesquereux-Martinez L, et al. A clinical model for predicting lymph node metastasis in submucosal invasive (T1) colorectal cancer. Int J Color Dis. 2015;30(6):761-8.

23. Taylor I, Mullee MA, Campbell MJ. Prognostic index for the development of liver metastases in patients with colorectal cancer. Br J Surg. 1990;77(5):499-501.

24. Segelman J, Akre O, Gustafsson UO, et al. Individualized prediction of risk of metachronous peritoneal carcinomatosis from colorectal cancer. Color Dis. 2014;16(5):359-67.

25. Huang $Y Q$, Liang $C H$, He $L$, et al. Development and validation of a radiomics nomogram for preoperative prediction of lymph node metastasis in colorectal cancer. J Clin Oncol. 2016;34(18):2157-64.

26. Hu T, Wang $S$, Huang $L$, et al. A clinical-radiomics nomogram for the preoperative prediction of lung metastasis in colorectal cancer patients with indeterminate pulmonary nodules. Eur Radiol. 2019;29(1):439-49.

27. Guan X, Ma CX, Quan JC, et al. A clinical model to predict the risk of synchronous bone metastasis in newly diagnosed colorectal cancer: a population-based study. BMC Cancer. 2019;19(1):704.

28. Oh JR, Park B, Lee $\mathrm{S}$, et al. Nomogram development and external validation for predicting the risk of lymph node metastasis in T1 colorectal cancer. Cancer Res Treat. 2019;51(4):1275-84.

29. Goossens-Beumer IJ, Derr RS, Buermans HP, et al. MicroRNA classifier and nomogram for metastasis prediction in colon cancer. Cancer Epidemiol Prevention Biomarkers. 2015;24(1):187-97.

30. Wang $X, C h i, P, L i n ~ H$, et al. Establishment of nomogram model to predict peritoneal metastasis in colon cancer patients without distant metastasis by 
preoperative imaging examination. Zhonghua Wei Chang Wai Ke Za Zhi. 2017;20(12):1387-92

31. Van Gijn W, Van Stiphout R, Van De Velde CJ, et al. Nomograms to predict survival and the risk for developing local or distant recurrence in patients with rectal cancer treated with optional short-term radiotherapy. Ann Oncol. 2015;26(5):928-35.

32. Valentini $V$, Van Stiphout RG, Lammering G, et al. Nomograms for predicting local recurrence, distant metastases, and overall survival for patients with locally advanced rectal cancer on the basis of European randomized clinical trials. J Clin Oncol. 2011;29(23):3163-72.

33. Sun $Y$, Lin $H$, Lu $X$, et al. A nomogram to predict distant metastasis after neoadjuvant chemoradiotherapy and radical surgery in patients with locally advanced rectal cancer. J Surg Oncol. 2017;115(4):462-9.

34. Hoshino N, Hasegawa S, Hida K, et al. Nomogram for predicting recurrence in stage II colorectal cancer. Acta Oncol. 2016:55(12):1414-7.

35. Renfro LA, Grothey A, Xue Y, et al. ACCENT-based web calculators to predict recurrence and overall survival in stage III colon cancer. J National Cancer Institute. 2014;106(12):dju333.

36. Tian X, Zhu X, Yan T, et al. Recurrence-associated gene signature optimizes recurrence-free survival prediction of colorectal cancer. Mol Oncol. 2017; 11(11):1544-60.

37. Peng J, Wang Z, Chen W, et al. Integration of genetic signature and TNM staging system for predicting the relapse of locally advanced colorectal cancer. Int J Color Dis. 2010;25(11):1277-85.

38. Ying HQ, Deng QW, He BS, et al. The prognostic value of preoperative NLR, d-NLR, PLR and LMR for predicting clinical outcome in surgical colorectal cancer patients. Med Oncol. 2014;31(12):305.

39. Zakaria S, Donohue JH, Que FG, et al. Hepatic resection for colorectal metastases: value for risk scoring systems? Ann Surg. 2007;246(2):183.

40. Kim C, Kim WR, Kim KY, et al. Predictive nomogram for recurrence of stage i colorectal cancer after curative resection. Clin Colorectal Cancer. 2018;17(3):e513-8.

41. Miyoshi N, Ohue M, Yasui M, et al. Novel prognostic prediction models for patients with stage IV colorectal cancer after concurrent curative resection. ESMO Open. 2016;1(3):e000052

42. Saso K, Myoshi N, Fujino S, et al. A novel prognostic prediction model for recurrence in patients with stage II colon cancer after curative resection. Mol Clin Oncol. 2018;9(6):697-701.

43. Hida K, Okamura R, Park SY, et al. A new prediction model for local recurrence after curative rectal cancer surgery: development and validation as an Asian collaborative study. Dis Colon Rectum. 2017:60(11):1168-74.

44. van Wyk HC, Roxburgh CS, Horgan PG, et al. The detection and role of lymphatic and blood vessel invasion in predicting survival in patients with node negative operable primary colorectal cancer. Crit Rev Oncol Hematol. 2014;90(1):77-90.

45. Stacker SA, Baldwin ME, Achen MG. The role of tumor lymphangiogenesis in metastatic spread. FASEB J. 2002;16(9):922-34

46. Bosch SL, Teerenstra S, de Wilt JH, et al. Predicting lymph node metastasis in PT1 colorectal cancer: a systematic review of risk factors providing rationale for therapy decisions. Endoscopy. 2013:45(10):827-41.

47. Longatto-Filho A, Pinheiro C, Ferreira $L$, et al. Peritumoural, but not intratumoural, lymphatic vessel density and invasion correlate with colorectal carcinoma poor-outcome markers. Virchows Arch. 2008;452(2):133-8.

48. Desolneux $G$, Burtin $P$, Lermite $E$, et al. Prognostic factors in node-negative colorectal cancer: a retrospective study from a prospective database. Int J Color Dis. 2010:25(7):829-34.

49. Matsumoto K, Nakayama $Y$, Inoue $Y$, et al. Lymphatic microvessel density is an independent prognostic factor in colorectal cancer. Dis Colon Rectum. 2007:50(3):308-14

50. Ha RK, Han KS, Sohn DK, et al. Histopathologic risk factors for lymph node metastasis in patients with T1 colorectal cancer. Ann Surg Treatment Res. 2017;93(5):266-71.

51. Yamauchi $H$, Togashi $K$, Kawamura $H$, et al. Tumor budding and pathological differentiation are predictive markers for lymph node metastasis in T1 colorectal cancer. Gastrointest Endosc. 2006;63(5): AB216.

52. Tateishi $Y$, Nakanishi $Y$, Taniguchi $H$, et al. Pathological prognostic factors predicting lymph node metastasis in submucosal invasive (T1) colorectal carcinoma. Mod Pathol. 2010;23(8):1068.

53. Van Wyk HC, Park J, Roxburgh C, et al. The role of tumour budding in predicting survival in patients with primary operable colorectal cancer: a systematic review. Cancer Treat Rev. 2015;41(2):151-9.
54. Glynne-Jones R, Wyrwicz L, Tiret E, et al. Rectal cancer: ESMO Clinical Practice Guidelines for diagnosis, treatment and follow-up. Ann Oncol. 2017; 28(suppl_4):iv22-40.

55. Knijn N, Mogk SC, Teerenstra S, et al. Perineural invasion is a strong prognostic factor in colorectal cancer. Am J Surg Pathol. 2016;40(1):103-12.

56. Benson AB, Venook AP, Cederquist $L$, et al. Colon cancer, version 1.2017, NCCN clinical practice guidelines in oncology. J Natl Compr Cancer Netw. 2017;15(3):370-98.

57. Liebig C, Ayala G, Wilks J, et al. Perineural invasion is an independent predictor of outcome in colorectal cancer. J Clin Oncol. 2009;27(31):5131.

58. Poeschl EM, Pollheimer MJ, Kornprat $\mathrm{P}$, et al. Perineural invasion: correlation with aggressive phenotype and independent prognostic variable in both colon and rectum cancer. J Clin Oncol. 2010;28(21):e358-60.

59. Peng J, Sheng W, Huang D, et al. Perineural invasion in pT3N0 rectal cancer: the incidence and its prognostic effect. Cancer. 2011;117(7):1415-21.

60. Huh JW, Kim HR, Kim YJ. Prognostic value of perineural invasion in patients with stage II colorectal cancer. Ann Surg Oncol. 2010;17(8):2066-72.

61. Taylor FG, Swift Rl, Blomqvist L, et al. A systematic approach to the interpretation of preoperative staging MRI for rectal cancer. Am J Roentgenol. 2008;191(6):1827-35.

62. Talbot IC, Ritchie S, Leighton MH, et al. The clinical significance of invasion of veins by rectal cancer. Br J Surg. 1980;67(6):439-42.

63. Günther K, Dworak O, Remke S, et al. Prediction of distant metastases after curative surgery for rectal cancer. J Surg Res. 2002;103(1):68-78.

64. Smith NJ, Barbachano Y, Norman AR, et al. Prognostic significance of magnetic resonance imaging-detected extramural vascular invasion in rectal cancer. Br J Surg. 2008;95(2):229-36.

65. Slesser AA, Khan F, Chau I, et al. The effect of a primary tumour resection on the progression of synchronous colorectal liver metastases: an exploratory study. Eur J Surg Oncol. 2015;41(4):484-92.

66. Hugen N, Van de Velde CJ, De Wilt JH, et al. Metastatic pattern in colorectal cancer is strongly influenced by histological subtype. Ann Oncol. 2014;25(3):651-7.

67. Grant MJ, Booth A. A typology of reviews: an analysis of 14 review types and associated methodologies. Health Information Libraries J. 2009;26(2):91108.

68. loannidis JP. The mass production of redundant, misleading, and conflicted systematic reviews and meta-analyses. Milbank Quarterly. 2016;94(3):485-514.

69. Greco T, Zangrillo A, Biondi-Zoccai G, Landoni G. Meta-analysis: pitfalls and hints. Heart Lung Vessel. 2013;5(4):219-25.

70. Bellou V, Belbasis L, Tzoulaki I, et al. Environmental risk factors and Parkinson's disease: an umbrella review of meta-analyses. Parkinsonism Relat Disord. 2016;23:1-9.

71. Theodoratou E, Tzoulaki I, Zgaga L, et al. Vitamin D and multiple health outcomes: umbrella review of systematic reviews and meta-analyses of observational studies and randomised trials. Bmj. 2014;348:g2035.

72. Belbasis L, Savvidou MD, Kanu C, et al. Birth weight in relation to health and disease in later life: an umbrella review of systematic reviews and metaanalyses. BMC Med. 2016;14(1):147.

\section{Publisher's Note}

Springer Nature remains neutral with regard to jurisdictional claims in published maps and institutional affiliations.

Ready to submit your research? Choose BMC and benefit from:

- fast, convenient online submission

- thorough peer review by experienced researchers in your field

- rapid publication on acceptance

- support for research data, including large and complex data types

- gold Open Access which fosters wider collaboration and increased citations

- maximum visibility for your research: over $100 \mathrm{M}$ website views per year

At $\mathrm{BMC}$, research is always in progress.

Learn more biomedcentral.com/submissions 